\title{
Organic halogen-bonded co-crystals for optoelectronic applications
}

\author{
Shuhai Chen ${ }^{1,3}$, Huiling Yin ${ }^{1}$, Jun-Jie Wu ${ }^{2}$, Hongtao Lin ${ }^{1 *}$ and Xue-Dong Wang ${ }^{2 *}$
}

\begin{abstract}
Organic halogen-bonded co-crystals assembled from two or more components via halogen bonds, have attracted significant attention due to their unpredictable and outstanding chemical and physical properties, which make them potential candidate materials for organic optoelectronic applications. This paper briefly summarizes the recent progress in terms of the developed fabrication methods for organic halogen-bonded co-crystals and their multifunctional optoelectronic applications. Based on the current research on organic halogen-bonded co-crystals, we further discuss the achievements as well as the existing challenges. Finally, we provide our outlooks for further studies and applications of organic halogen-bonded co-crystals.
\end{abstract}

Keywords: organic semiconductor molecules, single crystals, self-assembly, non-covalent interaction, organic optoelectronic

\section{INTRODUCTION}

The synthesis of organic co-crystals has been recognized as an efficient approach for designing functional organic solids, for the applications in organic field-effect transistors [1-4], organic light-emitting diodes [5-7], organic solid-state lasers [8-11], optical waveguides [12-16], organic light-emitting transistors [17-20], and sensors [2123]. Organic co-crystals comprise at least two types of components formed through noncovalent intermolecular interactions, such as $\pi-\pi$ stacking interaction, chargetransfer (CT) interaction, hydrogen bonds (HBs), and halogen bonds (XBs) [24-26]. Noncovalent interactions regulate the molecular packing pattern and crystallinity of organic solid-state materials, which, in turn, affect their optoelectronics performance [27-30]. Especially, halogen bonds are regarded as highly oriented bonds. They give rise to intermolecular transport of electrons [31], which is a thriving strategy for designing multifunctional and high-performance optoelectronic organic solid-state materials [32-35]. The International Union of Pure and Applied Chemistry (IUPAC) defines the term "halogen bonding" as follows: "it occurs when there is evidence of a net attractive interaction between an electrophilic region associated with a halogen atom in a molecular entity and a nucleophilic region in another, or the same, molecular entity" [36]. As shown in Scheme 1, an HB can be represented by R-X...Y (the three dots denote the bond), where $\mathrm{X}$ is a halogen atom with an electrophilic area with which it covalently attaches to $\mathrm{R}$; $\mathrm{R}-\mathrm{X}$ denotes the $\mathrm{XB}$ donor; and $\mathrm{Y}$ is the $\mathrm{XB}$ acceptor with a nucleophilic area, which can be an anion or a neutral component $[37,38]$.

In 1814, Colin [39] discovered the adducts between iodine and ammonia. In 1863, Guthrie et al. [40] purified and formulated the above adducts as a $\mathrm{NH}_{3} \cdot \mathrm{I}_{2}$ complex. In 1954, Hassel et al. [41], for the first time, described the solid-state structure of $\mathrm{Br}$... $\mathrm{O}$ short-distance contact (2.71 $\AA$ ) between dibromine and 1,4-dioxane through the crystal X-ray diffraction (XRD) experiment, and confirmed the existence of electrostatic attraction, which was a landmark in the understanding of the XB [42]. In 1968, Bent [43] verified that the distance between the halogen atom and the electron donor atom was smaller than the sum of their respective van der Waals radii, and in energy

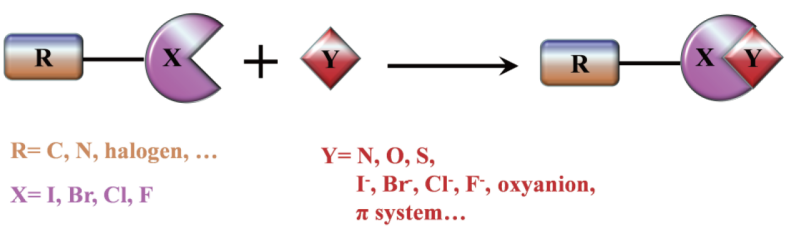

Scheme 1 Schematic diagram of halogen bond.

\footnotetext{
${ }^{1}$ School of Chemistry and Chemical Engineering, Shandong University of Technology, Zibo 255000, China

2 Jiangsu Key Laboratory for Carbon-Based Functional Materials \& Devices, Institute of Functional Nano \& Soft Materials (FUNSOM), Soochow University, Suzhou 215123, China

${ }^{3}$ School of Chemical Engineering, Qingdao University of Science \& Technology, Qingdao 266044, China

* Corresponding authors (emails: wangxuedong@suda.edu.cn (Wang XD); linht@sdut.edu.cn (Lin H))
} 
the interaction was equivalent to a strong HB. Clark et al. [44], through theoretical calculations, discovered that the electron density surrounding a XB was anisotropic in distribution, and thus, proposed the concept of $\sigma$-hole. In 1998, Resnati et al. [45,46] reported the tendency of iodine and bromoperfluorocarbons to form noncovalent interactions with neutral and anionic nitrogen as electron donors [47]. In addition, they indicated that the XB interaction force can be regulated by altering the halogen atoms and the covalently bonded groups [48]. Resnai et al. $[49,50]$ also demonstrated that the strongest complex can be formed between nucleophilic nitrogen atoms and iodine atoms, whose electrophilicity can be enhanced by covalently bonding to an electron-poor perfluorocarbon group. When the environment around the halogen is sufficiently electron-withdrawing, the resulting intermolecular interaction will become strong enough to affect the crystal packing in the organic solid [51,52]. Meanwhile, there are many reports on the theoretical calculations of XBs $[53,54,55]$. For example, Wang et al. [54] investigated a series of blue-shifting halogen-bonded complexes, through which they further confirmed the CT interactions in XBs and noted that the large dipole moments of interacting monomers were important for multiple halogen bonding systems.

Currently, halogen bonding is widely employed as an effective strategy by crystal engineers for designing functional solid-state architectures [56-58]. However, the challenge in the syntheses of organic halogen-bonded cocrystals lies in the selection of the co-formers and regulation of the molecular packing mode $[59,60]$. Until now, stilbene derivatives and cyanostilbene-based positional isomers, such as 1,4-bis (4-cyanostyryl) benzene ( $p$ BCB), 1,2-bis(4-pyridy)ethylene (Bpe), 4-(1-naphthylvinyl)pyridine (DPEpe), (Npe)4,4'-((1E,1E)-2,5-dimethoxy-1,4-phenylene)bis(ethene-2,1-diyl))dipyridine, 4-styrylpyridine (Spe), and (E)-2-(((5-methoxypyridin-2yl)imino)methyl)phenol (MPI), are commonly used as halogen acceptors (Scheme 2a) [7,61,62]. Besides, fluorine and perfluorinated co-formers, such as 1,3,5-trifluoro2,4,6-triiodobenzene (IFB) and 1,4-diiodotetrafluoro benzene (F4DIB), are commonly employed as halogen donors, which promotes the tendency of the iodine atoms to behave as electron acceptors in $\mathrm{XB}$ networks (Scheme 2b) [63-65].

The types of halogen donors and acceptors modulate the stacking patterns of organic co-crystals, which affect the properties of the co-crystals. Thus, an urgent problem associated with organic halogen-bonded co-crystals is to broaden the molecular systems. However, XBs have been confirmed to regulate the photoluminescence (PL) and intermolecular electron transport in organic co-crystals. Thus, organic co-crystals do not simply combine the properties of the constituent molecules, which usually

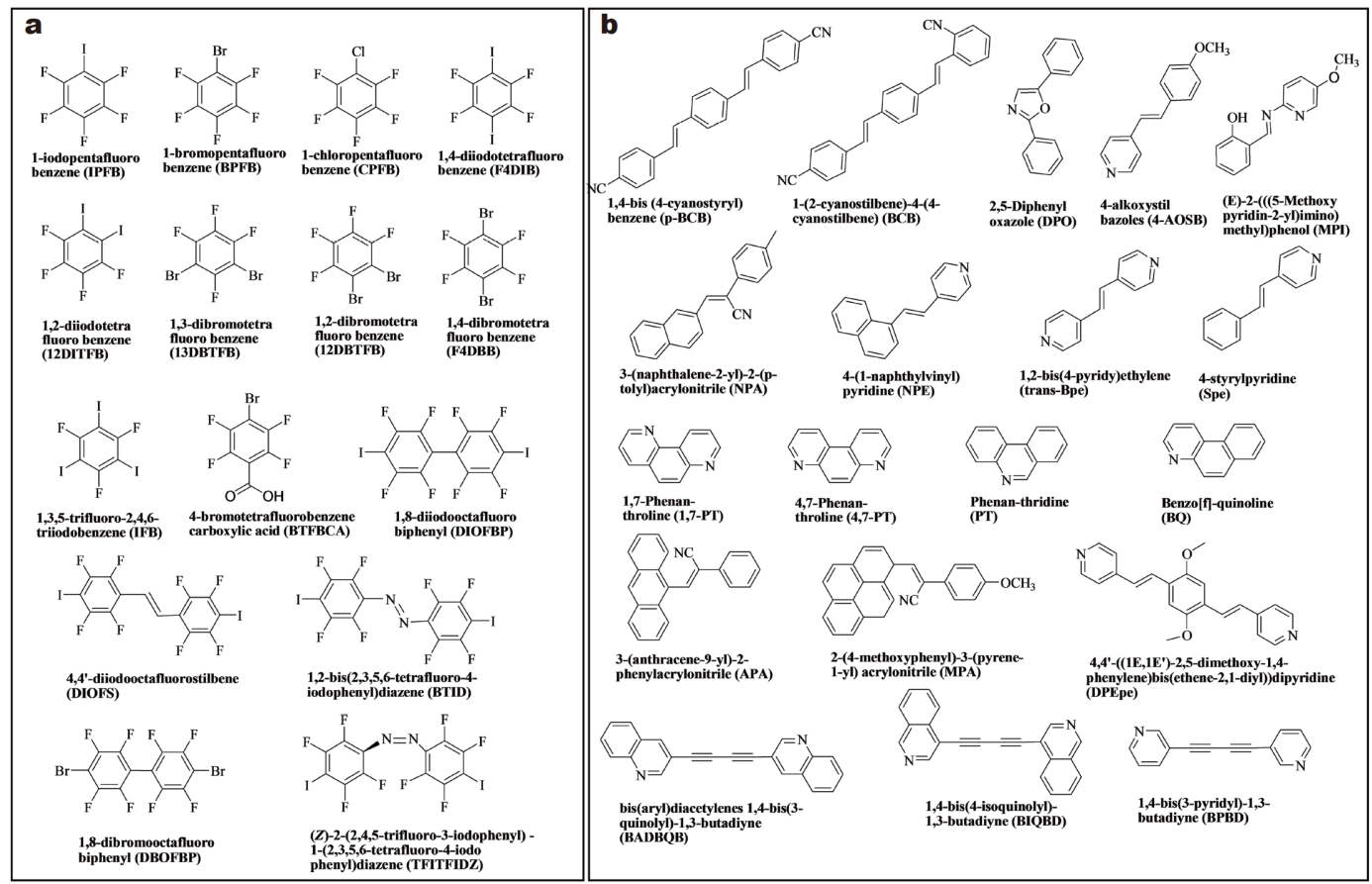

Scheme 2 Structures of the typical (a) halogen donors and (b) halogen acceptors applied in organic halogen-bonded co-crystals. 
exhibit unique optoelectronic properties. To date, most reported studies have focused on modulating the optical and photoelectronic properties of organic halogen-bonded co-crystals $[17,50,66,67]$. On one hand, owing to the heavy-atom effect, the organic halogen-bonded co-crystals can be promising phosphorescent materials. Bolton et al. [68] first fabricated bright and color-tunable organic phosphors by taking advantage of the heavy-atom effect of XBs, and recorded a PL quantum efficiency of up to $55 \%$. On the other hand, XB-based co-crystals can also be used to tune the fluorescence properties of solid-state materials. Yan et al. [69] designed and fabricated a series of organic co-crystals employing a stilbene-type derivative, 1,4-bis-p-cyanostyrylbenzene, to form co-crystals with perfluorocarbon compounds, which were commonly used as XB donors. By introducing co-formers, a series of co-crystals with distinct crystal structures were obtained, which exhibited multi-color emission besides strong twophoton luminescence. These phenomena were attributed to the difference between the geometric configurations of the co-crystals, which influenced the formation of the excimers in the organic co-crystals. This demonstrated a new insight into the structure-performance relation of organic co-crystals. Also, we have realized the regulation of the crystallinity and optical properties of organic halogen-bonded co-crystals, which is expected to prompt the development of organic integrated optoelectronic devices [70].

Recently, despite many excellent reported reviews on organic co-crystals $[58,71,72]$, only a few have focused on the organic halogen-bonded co-crystals. In this review, we summarize various reported studies that focus on the fabrication strategies and optoelectronic applications of organic halogen-bonded co-crystals. We also provide perspectives for further investigations on organic halogen-bonded co-crystals and their potential applications in high-performance organic optoelectronic devices.

\section{PREPARATION STRATEGIES}

The main strategies for synthesizing organic halogenbonded co-crystals include solution evaporation, mechanochemical, and liquid-assisted grinding methods. The preparation methods significantly affect the stacking pattern and crystal morphology, which in turn, affect the optical and optoelectronic properties of the organic cocrystals.

\section{Solution evaporation}

Currently, the solution evaporation method is a universal method for fabricating organic co-crystals with low defect and uniform morphology, which are desired for further optoelectronic characterization $[6,73,74]$. Meanwhile, the solution evaporation method is greatly affected by external conditions such as the type and proportion of the solvent, concentration of the precursor, and the temperature of crystallization. For instance, the microcrystals of DPEpe-F4DIB with different one-dimensional (1D) structures can be controllably synthesized via simple solvent evaporation based on different solvent systems, as shown in Fig. 1 [75]. The precursors of DPEpe and F4DIB
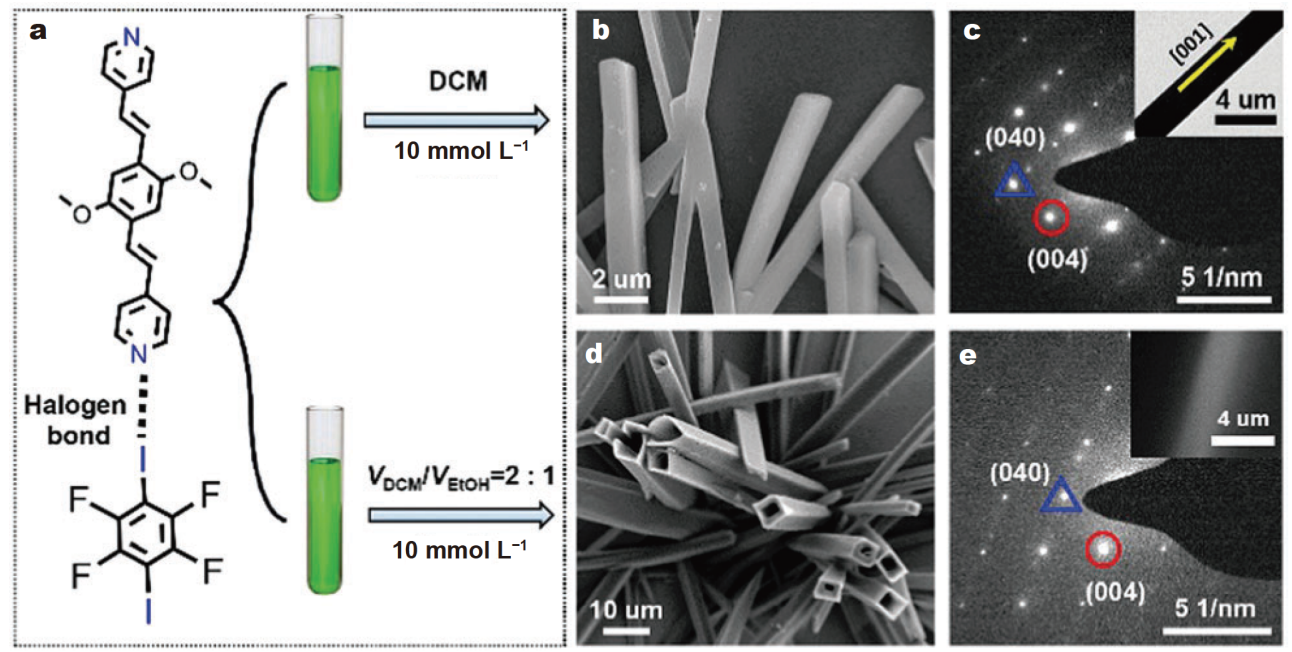

Figure 1 (a) Fabrication procedure for DPEpe-F4DIB microcrystals by solution-evaporation method. (b) SEM image of the DPEpe-F4DIB microrods. (c) SAED pattern of the DPEpe-F4DIB microrods. (d) SEM image of the DPEpe-F4DIB microtubes. (e) SAED pattern of the DPEpe-F4DIB microtubes. The inset: transmission electron microscopy (TEM) images. Reprinted with permission from Ref. [75]. Copyright 2018, Royal Society of Chemistry. 
are bonded via the XBs between nitrogen and iodine (Fig. 1a). The procedure is as follows: DPEpe and F4DIB are first dissolved in dichloromethane (DCM), and then dropped directly onto a quartz substrate to obtain $1 \mathrm{D}$ cocrystal microrods, with a smooth surface and uniform diameter, after complete evaporation of the solvent (Fig. 1b). The scanning electron microscopy (SEM) image and the corresponding selected area electron diffraction (SAED) pattern indicate that the DPEpe-F4DIB co-crystal microrods belong to the triclinic space group, which grows along the [001] direction (Fig. 1c, d). In contrast, when DPEpe and F4DIB are dissolved in a mixed solvent of DCM and ethanol $\left(V_{\mathrm{DCM}}: V_{\text {ethanol }}=2: 1\right)$, the above procedure yields $1 \mathrm{D}$ microtubes (Fig. 1d). The microtubes follow the same crystal growth direction as that of the organic microrods (Fig. 1d). In addition, the obtained organic microrods in pure DCM can evolve into microtubes in the abovementioned solvent mixture, which is attributable to the etching effect of ethanol. The obtained molecular packing structures of DPEpe-F4DIB indicate that the molecules are separated and stacked along the $c$ axis, which facilitates the formation of 1D morphology. This paper proposes a beneficial strategy for a simple fabrication of multifunctional organic co-crystal optoelectronic devices based on identical molecule systems.

With the facile solvent evaporation method, we mod- ulate the yellow-emissive $p$-BCB polyhedral microcrystals to the blue-emissive $p$-BCB.DIFB co-crystals formed by using XBs (Fig. 2a, c) [70]. The typical synthesis procedure involves dissolving $p$-BCB and DIFB in chloroform in a molar ratio of $1: 1$, and then, dropping the solution onto a substrate. The XRD pattern demonstrates that the $p$-BCB.DIFB co-crystal belongs to the monoclinic system, whereas the $p-\mathrm{BCB}$ organic microwire belongs to the triclinic system. In addition, the intermolecular distance of $p$-BCB is $0.71 \mathrm{~nm}$ in the $p$-BCB.DIFB co-crystals, which is much larger than the required distance for the excimer formation $(0.35 \mathrm{~nm})$. Comparing the PL spectra of $p$-BCB microcrystals with that of $p$-BCB.DIFB cocrystals, it is found that the orange emission of the $p-\mathrm{BCB}$ microcrystals turns into blue emission of $p$-BCB.DIFB cocrystals, and the PL spectra of the co-crystals exhibit a fine electronic-vibrotational structure (Fig. 2d). This indicates that the growth morphology and light emission can be modulated via the co-crystal strategy.

\section{Mechanochemical approach}

In the solution method, the components are required to have similar solubility. In comparison, the mechanochemical approach does not involve the solvent, and thus, can be easily performed. It has been demonstrated to be a clean, green, and effective method for the synth- a
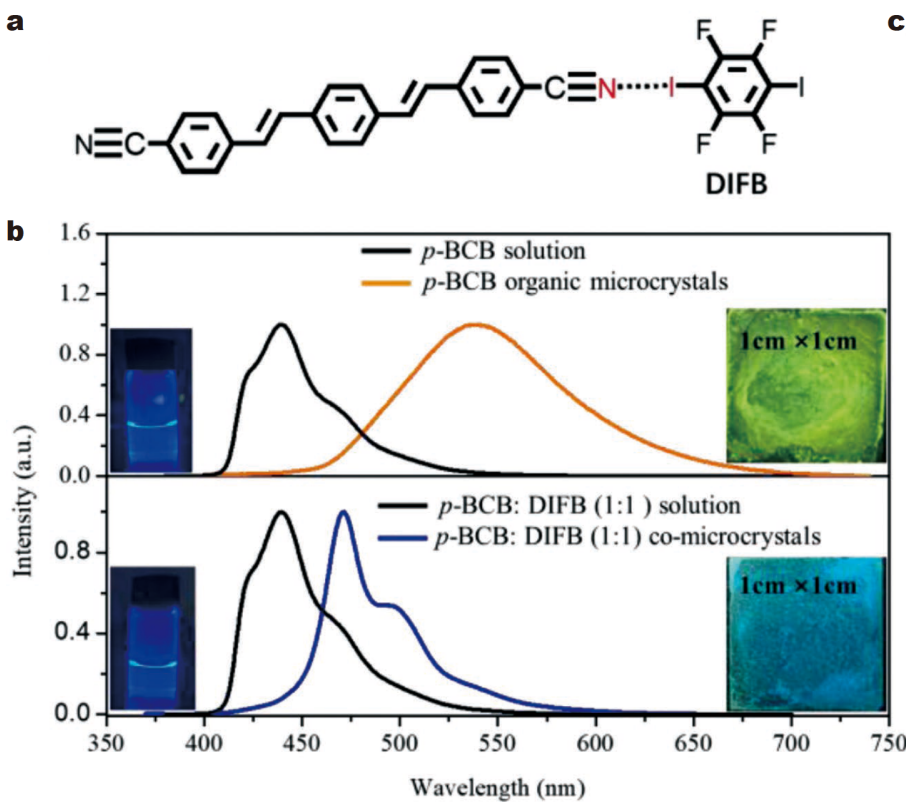
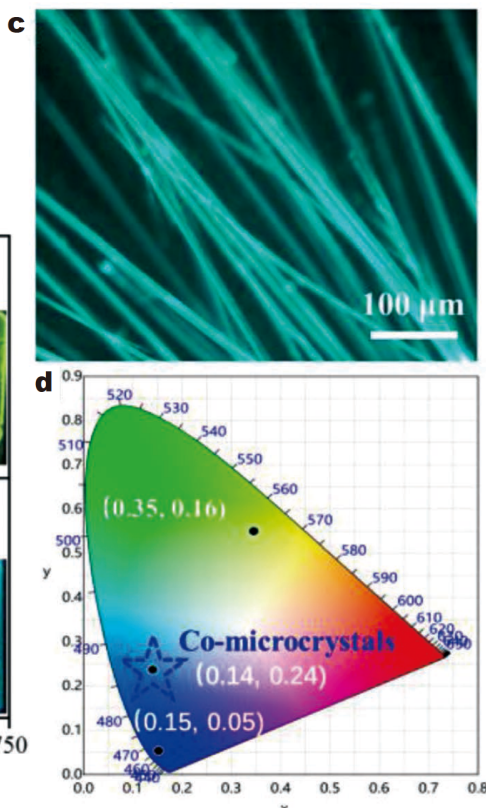

Figure 2 (a) The schematic of halogen bond force between $p$-BCB and DIFB molecule. (b) The PL spectra of $p$-BCB solution and organic microcrystals (top panel) and the $p$-BCB and DIFB mixed solution and $p$-BCB.DIFB co-crystals (bottom panel). Inset: the photographs of $p$-BCB microcrystals (top panel) and $p$-BCB-DIFB co-crystals (bottom panel) under UV lamp. (c) Fluorescence microscopy image of $p$-BCB.DIFB co-crystals. (d) The CIE chromaticity diagram of the co-crystals and solution. Reprinted with permission from Ref. [70]. Copyright 2018, Wiley-VCH. 
a

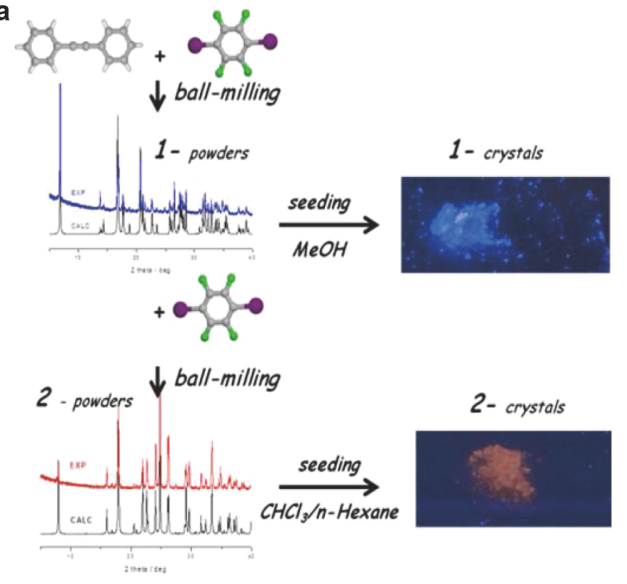

b

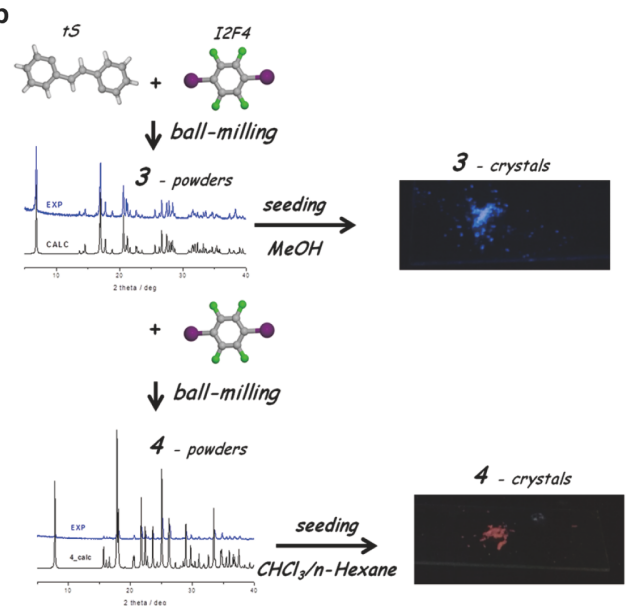

Figure 3 Synthetic schematic and the corresponding fluorescence photographs of co-crystals (a) DPA [DPA. $\mathrm{I}_{2} \mathrm{~F}_{4}$ (1) and DPA. $\left(\mathrm{I}_{2} \mathrm{~F}_{4}\right)_{2}(2)$, (b) $\mathrm{tS} \cdot \mathrm{I}_{2} \mathrm{~F}_{4}(3)$ and $\mathrm{tS} \cdot\left(\mathrm{I}_{2} \mathrm{~F}_{4}\right)_{2}(4)$. Reprinted with permission from Ref. [77]. Copyright 2015, American Chemical Society.

eses of organic halogen-bonded co-crystals [72,76-78]. For instance, d'Agostino et al. [77] synthesized four types of organic co-crystals, denoted as DPA.I2F4 (1), DPA.(I2F4) ${ }_{2}$ (2), tS.I2F4 (3), and tS.(I2F4) ${ }_{2}$ (4) via the mechanochemical method. The procedure for the cocrystal synthesis was as follows: I2F4, DPA, or tS were ground in a molar ratio of 1:1, and the obtained products were divided into two portions (Fig. 3). One portion was used for single crystal growth by seeding in methanol (1crystals), while the other portion further reacted with an additional equal amount of I2F4 co-former. The freshly obtained solid product was grown into single crystals by seeding in a mixture of solvents $\left(\mathrm{CHCl}_{3} / n\right.$-hexane) (2crystals). I2F4 and tS were subjected to the same treatments as those stated above, to obtain two other single crystals (3-crystals and 4-crystals). As shown in Fig. 3a, b, the 1- and 3-crystals, with a co-former molar ratio of 1:1, are blue-emissive crystals, exhibiting fluorescence and phosphorescence emission properties. Meanwhile, the other two co-crystals with a molar ratio of 1:2 exhibit reddish-brown emission crystals, which are verified to be triplet emitters with the highest phosphorescence quantum efficiency of 0.06 at room temperature.

From the X-ray analysis of the single-crystal structures, it is found that the DPA. $\mathrm{I}_{2} \mathrm{~F}_{4}(1)$ and $\mathrm{tS} \cdot \mathrm{I}_{2} \mathrm{~F}_{4}$ (3) co-crystals are intercalation solids. The co-former of the I2F4 molecule is almost perpendicular to the benzene ring in an alternating consecutiveness in the 1D structure of DPA or $\mathrm{tS}$, where the $\mathrm{C}-\mathrm{I} \cdots \pi$ interactions play a more important role than $\mathrm{C}-\mathrm{H} \cdots \pi$ interactions in pure DPA and tS crystals (Fig. 4). As shown in Fig. 5, DPA. $\left(\mathrm{I}_{2} \mathrm{~F}_{4}\right)_{2}(2)$ and $\mathrm{tS} \cdot\left(\mathrm{I}_{2} \mathrm{~F}_{4}\right)_{2}$ (4) are arranged in a $2 \mathrm{D}$ network. However, a
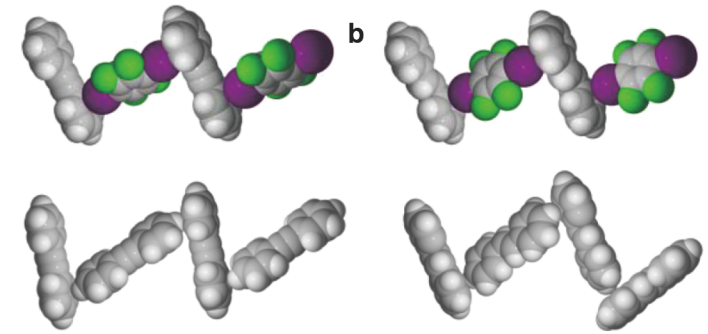

Figure 4 Space filling representation of (a) crystalline 1 and (b) crystalline 3. Reprinted with permission from Ref. [77]. Copyright 2015, American Chemical Society.
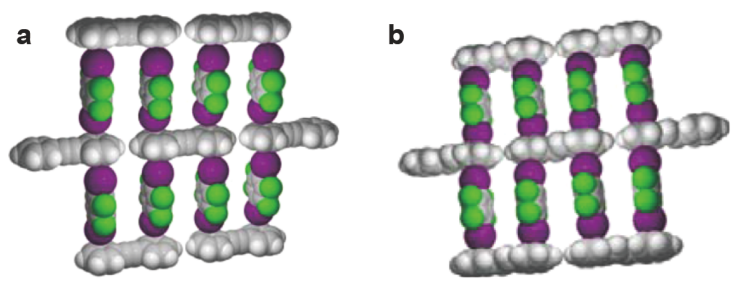

Figure 5 Space filling representation of (a) crystalline 2 and (b) 4 . Reprinted with permission from Ref. [77]. Copyright 2015, American Chemical Society.

attempts to synthesize the above co-crystals via the solution method are unsuccessful. This mechanochemical method provides a new strategy for regulating the photophysical properties of organic co-crystals by varying the stoichiometric ratio of the halogen donor and acceptor.

\section{Liquid-assisted grinding approach}

The liquid-assisted grinding method is considered to be superior to the solvent-free grinding method, which 


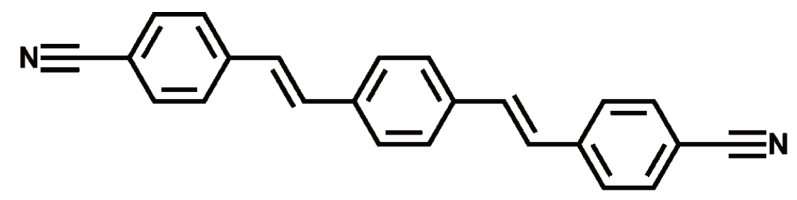

1,4-bis-p-cyanostyrylbenzene (A)

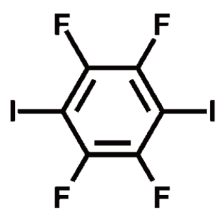

1,4-diiodotetrafluorobenzene (B)<smiles>Fc1c(F)c(Br)c(F)c(F)c1Br</smiles>

1,4-dibromotetrafluorobenzene (E)

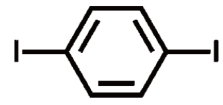

1,4-diiodobenzene (C)

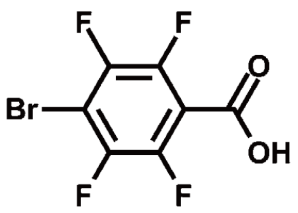

4-bromotetrafluorobenzene carboxylic acid (F)

Scheme 3 Chemical formula of the halogen acceptor (A) and halogen donors (B, C, D, E, F, G). Reprinted with permission from Ref. [69]. Copyright 2011, Wiley-VCH.

usually exhibits higher yields, better crystallinity, less likely deformation, and wider option scope of precursors $[79,80]$. For example, Yan et al. [69] synthesized six types of co-crystals via the liquid-assisted grinding strategy with 1,4-bis-p-cyanostyrylbenzene (A) as the fluorescent model system and compounds with a weak visible solidstate fluorescence as the co-formers (Scheme 3). However, co-crystals cannot be directly obtained via co-crystallization from the solution due to the difference in the solubilities of the co-formers. The co-formers B, C, E, F, and $\mathrm{G}$ afford functional groups commonly used in supramolecular synthesis, which can be assembled into $1 \mathrm{D}$ chain structures with $\mathrm{A}$ via $\mathrm{XB}$ or $\mathrm{HB}$ interactions. In addition, single crystals can be obtained via crystallization, by slowly evaporating the solvent from the chloroform solution of the co-crystal.

Observing the emission of co-crystals 1-6, their color under daylight illumination is different from that of pure A crystals, as shown in Fig. 6a. When irradiated with ultraviolet light, these co-crystals are found to exhibit multicolor luminescence, changing from blue to green, and to yellow (Fig. 6b). Fig. 6d shows the fluorescence images of the transparent co-crystals 1-6, where the luminescence of the single crystals is similar to that of the powder, whereas the co-crystals exhibit tunable emission (Fig. 6c). The two-photon emission of the prepared cocrystals at a single wavelength of $800 \mathrm{~nm}$ laser can be tuned to emit light from blue to yellow, and hence, it is a candidate material for two-photon fluorescence. It is concluded that the liquid-assisted grinding method offers more possibilities for designing co-crystals, which have potential applications in luminescent materials.

\section{OPTOELECTRONIC APPLICATIONS}

\section{Optical waveguide: 1D photon transport}

Optical waveguides based on organic halogen-bonded cocrystals, especially the 1D organic solid-state materials,

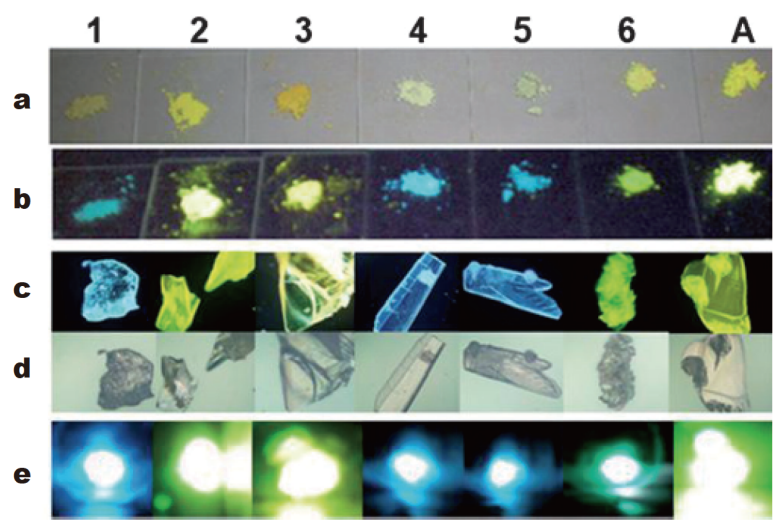

Figure 6 Photographs of (a) the co-crystal samples (1-6) and A. (b) The corresponding powder under sunlight and $365 \mathrm{~nm}$ UV light. (c, d) The fluorescence microscope of co-crystal samples (1-6) and A under 365 nm UV and sunlight. (e) Two photon luminescence under $800 \mathrm{~nm}$ laser. Reprinted with permission from Ref. [69]. Copyright 2011, Wiley-VCH. 
have recently attracted research interest due to their potential applications in organic photonic devices, sensors, light-concentrating devices, and waveguides [81-85]

As reported earlier [75], 1D microrods and microtubes of DPEpe-F4DIB co-crystals can be controllably fabricated via the solution-evaporation method by tuning the solvent system. The optical waveguide features of the asprepared 1D microrod and microtube co-crystals were characterized to determine the structure-property relation for the micro/nanostructures. According to the micro-area fluorescence microscopy image of the asprepared DPEpe-F4DIB microrods and microtubes excited at different positions with a $375 \mathrm{~nm}$ laser beam, the photons are restricted in two dimensions and travel in two opposite directions along the central axis of the microrod (Fig. 7a, c). The spatially resolved PL spectra record the fluorescence intensity in the excitation site along the 1D microcrystal $\left(I_{\text {body }}\right)$ and the tip of the luminescence $\left(I_{\text {tip }}\right)$ (Fig. $\left.7 \mathrm{~b}, \mathrm{~d}\right)$. A single exponential decay relation is observed for $I_{\text {tip }} / I_{\text {body }}$ (Fig. 7e), which indicates active waveguide properties. The optical-loss coefficients $(R)$ for the microrods and microtubes are 0.0341 and $0.0145 \mathrm{~dB} \mathrm{\mu m}^{-1}$ at $525 \mathrm{~nm}$, respectively, according to the equation $I_{\text {tip }} / I_{\text {body }}=A \exp (-R D)$. Compared with the previous reports, the $R$ value is low enough for organic optical waveguides, which indicates fine photonic transmission property. In addition, the much lower opticalloss coefficient of the organic co-crystal microtubes, as compared with that of the microrods, can be attributed to the following: i) the hollow microtubes, which form circular microcavity, exhibit more efficient confinement effect on the photons than that of the microrods, due to the much smaller refractive index of air $\left(n_{\text {air }}=1\right)$, compared with that of the $1 \mathrm{D}$ organic crystal $\left(n_{\text {crystal }} \approx 2\right)$; ii) the defect traps inside the organic microrods lead to much more optical loss as the light transmits along the axis of the organic microrods, which does not exist in the organic microtube; iii) the thin tubular walls of the microtubes also contribute to their much lower optical loss. These 1D organic co-crystals are promising building
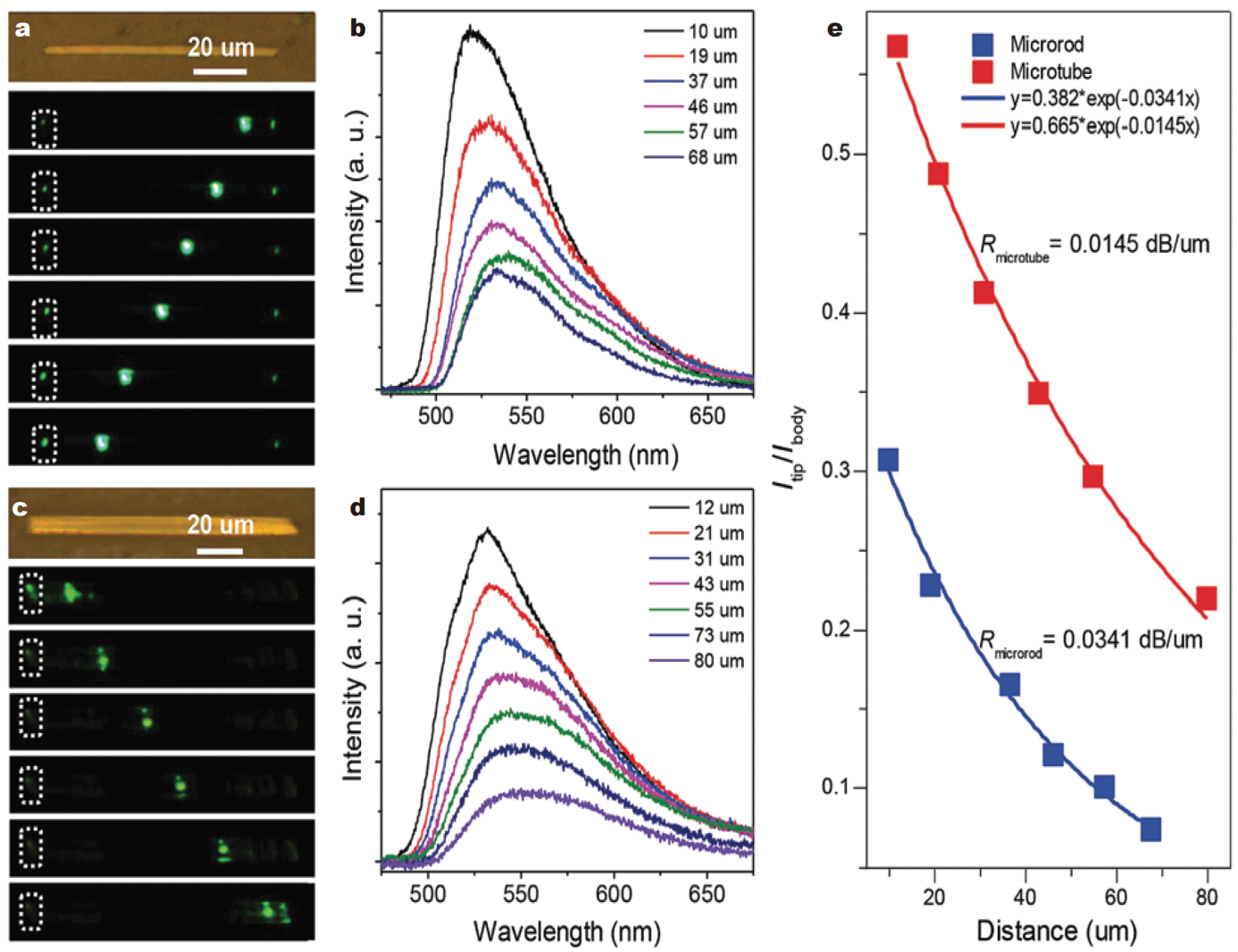

Figure 7 (a, c) Bright-field and fluorescence micrograph images of a single DPEpe-F4DIB microrod and microtube excited at different sites with 375 $\mathrm{nm}$ laser beam. (b, d) Spatially resolved PL spectra from the tip of the microrod and microtube for different separation distances between the excitation site and the tip as shown in (a) and (c). (e) Ratios of $I_{\text {tip }} / I_{\text {body }}$ and the distance $D$ corresponding to (a) and (c). Reprinted with permission from Ref. [75]. Copyright 2018, Royal Society of Chemistry. 
blocks for optical waveguide devices.

\section{Optical waveguide: 2D photon transport}

Few single-component organic molecules exhibit 2D selfassembly behavior, which greatly impedes the progress of 2D organic photonics and electronics [86]. Compared with 1D organic crystals, 2D organic crystals with good morphology exhibit excellent photon-confinement effect, which makes them more appropriate for the applications in a microscale planar photonics system [87-89]. Thus, by choosing appropriate co-formers, organic co-crystals held together by anisotropic XBs can be ideal candidates for achieving optoelectronic properties that cannot be achieved with a single component [89].

We have previously synthesized regular $2 \mathrm{D}$ organic halogen co-crystals using DPEpe and F4DIB molecules (Fig. 8) [90]. These as-prepared 2D organic co-crystals exhibit asymmetric light propagation. To reveal the asymmetric transmission in the DPEpe-F4DIB 2D cocrystal, the properties of the optical waveguide were investigated. The results reveal symmetrical and asymmetric optical waveguides in two directions. The optical waveguides along the [100] direction are symmetrical, and exhibit similar optical loss coefficients $\left(R_{\text {Upward }}=\right.$ $0.0344 \mathrm{~dB} \mathrm{\mu m}^{-1}$ and $R_{\text {Downward }}=0.0333 \mathrm{~dB} \mathrm{\mu m}^{-1}$ ) (Fig. 9ad). Interestingly, asymmetric optical waveguides along the [010] direction are also observed and the optical loss coefficients are $R_{\text {Backward }}=0.0346 \mathrm{~dB}_{\mu} \mathrm{m}^{-1}$ and $R_{\text {Forward }}=$ $0.0894 \mathrm{~dB} \mu \mathrm{m}^{-1}$. The difference in the optical loss coefficients is attributed to an anisotropic molecular stacking pattern of the 2D DPEpe-F4DIB co-crystals (Fig. 9e-h). We anticipate that these $2 \mathrm{D}$ organic co-crystals with asymmetric photon transport can be applied in optical logic gates and other organic photonics at micro/na-

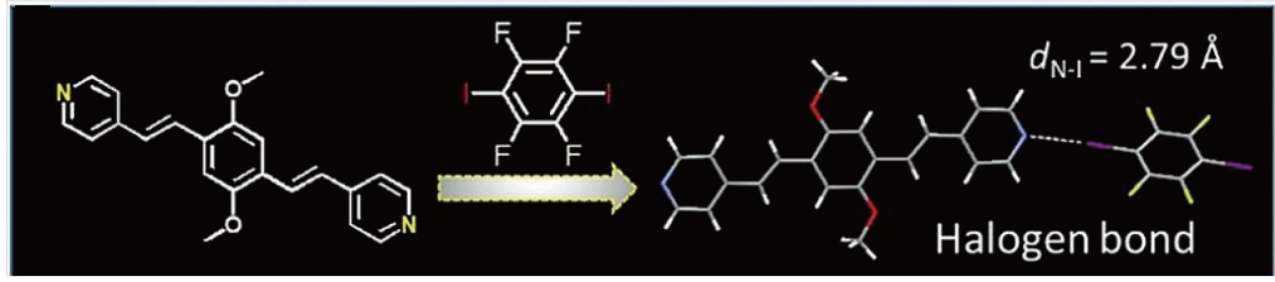

Figure 8 The chemical structure of DPEpe-F4DIB halogen-bonded co-crystal. Reprinted with permission from Ref. [90]. Copyright 2018, Wiley$\mathrm{VCH}$.
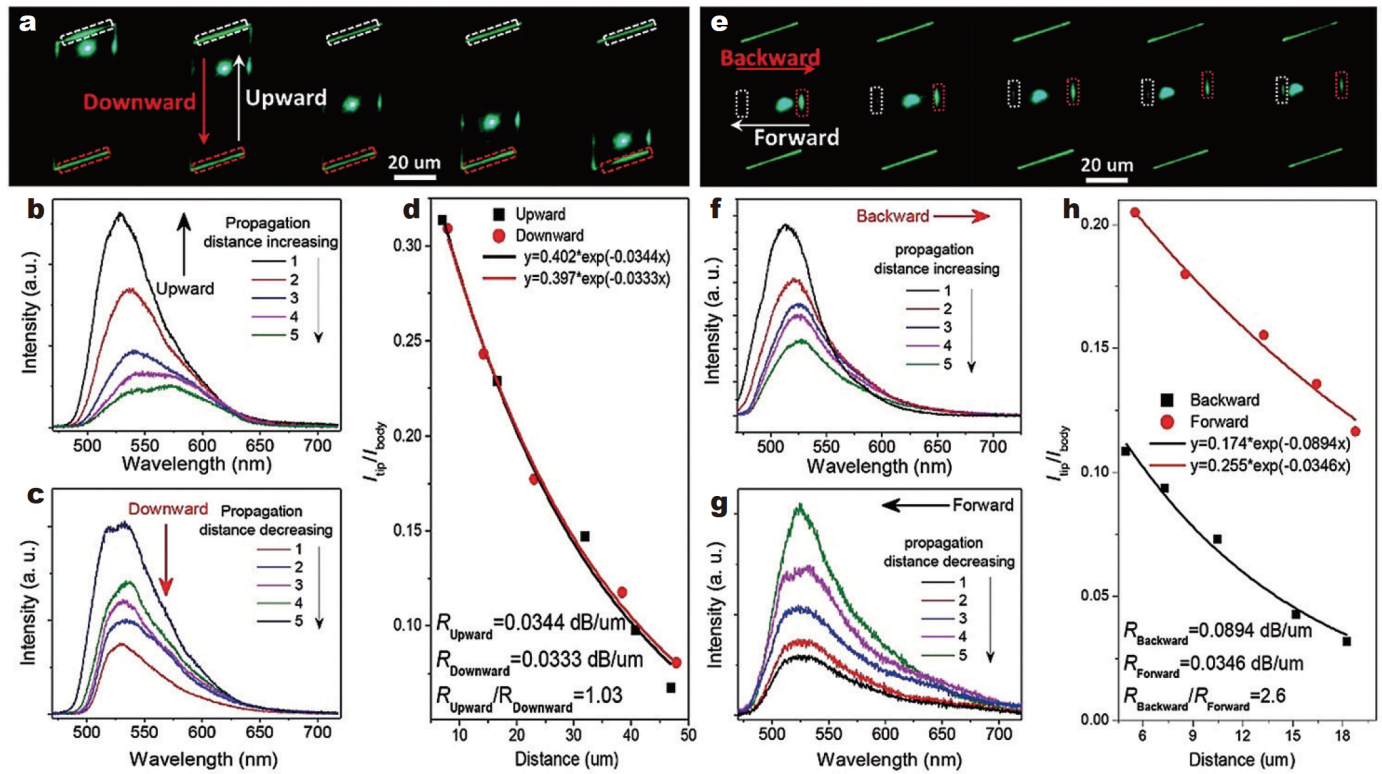

Figure 9 (a, e) Fluorescence microscopy images of DPEpe-F4DIB co-crystal excited by $375 \mathrm{~nm}$ laser at different site along with [100] and [010] direction, respectively. (b, c) and (f, g) The corresponding spatially resolved PL spectra in (a) and (e). (d, h) Ratios of $I_{\text {tip }} / I_{\text {body }}$ and the distance $D$ corresponding to (a) and (e). Reprinted with permission from Ref. [90]. Copyright 2018, Wiley-VCH. 
noscale.

\section{Organic solid-state laser}

Organic solid-state lasers (OSSLs) have received substantial attention due to their potential application in some frontier optoelectronics [91-95]. Although the gained molecular systems that can be used in OSSLs are very limited, organic co-crystals broaden the range of options [96,97]. For instance, Chu et al. [98] designed the first organic halogen-bonded co-crystal microlaser using MPI as the halogen acceptor and F4DIB and IFB as the halogen donors. As shown in Fig. 10a, MPI can be selfassembled into a $1 \mathrm{D}$ structure via $\pi-\pi$ stacking along the $c$-axis. However, the insertion of F4DIB molecules induces the formation of a $2 \mathrm{D}$ plate-like structure along the $a$-axis, as well as $\pi \cdots \mathrm{O}$ interactions, with the aid of the $\mathrm{O} \cdots \mathrm{H}$ bond (Fig. 10b). The formation of the $2 \mathrm{D}$ morphology co-crystal MPI.F4DIB is attributed to the strong halogen bond of $\mathrm{N} \cdots \mathrm{I}$ and $\mathrm{I} \cdots \pi$ in the $b c$-plane between the MPI and F4DIB molecules (Fig. 10c). The CT between the $\mathrm{N}$ and iodine atoms among the halogen bond interactions makes the co-crystals potential candidates for stimulated emissions. To regulate the energy band and the molecular packing model of the co-crystal, IFB having one more iodine atom in its skeleton than F4DIB is used as the halogen bond donor. IFB is expected to have a stronger intermolecular interaction with the halogen bond acceptor, MPI, than F4DIB, which results in the formation of a ring-like MIC co-crystal (Fig. 10d). In the MIC co-crystals, the MPI and IFB molecules are held together by XBs, such as $\mathrm{I} \cdots \pi, \mathrm{N} \cdots \mathrm{I}$, and $\mathrm{I} \cdots \mathrm{O}$, which provides a more efficient $\mathrm{CT}$ in the MIC co-crystal than that in the MFC co-crystal (Fig. 10d).

To investigate the laser performance of the MPI cocrystal microwire and MFC microplate, optical-pump laser measurements were performed. The MPI microwire shows green emission at its terminal while being excited by a $400 \mathrm{~nm}$ pulsed laser (inset in Fig. 11a). A sharp cavity-mode peak located at $\sim 560 \mathrm{~nm}$ is also observed, as shown in the PL spectra in Fig. 11a. The specific knee signal at $116.5 \mu \mathrm{J} \mathrm{cm}^{-2}$, as shown in Fig. $11 \mathrm{~b}$, indicates the laser behavior for the MPI microwire. As the pump fluence increases, the full width at half maximum (fwhm) at $551 \mathrm{~nm}$ becomes as narrow as $0.24 \mathrm{~nm}$ (Fig. 11b), which indicates distinct microcavity effects, and the $Q$ (defined as $\lambda / f w h m$ ) value becomes as high as 2300 . Moreover, the linear correlation between $\lambda^{2} / 2 \Delta \lambda$ and $L$, as shown in Fig. 11c, demonstrates a classical Fabry-Pérot-type resonance. The antinodes are confirmed by characterizing the electromagnetic field intensity distributions (inset in Fig. 11c). In addition, owing to the regular 2D defect-free facets, a self-waveguided edge emission for the MFI cocrystal microplate is observed, which is considered to be of great importance in light confinement. When the pump fluence is increased to over $12.74 \mu \mathrm{J} \mathrm{cm}^{-2}$, the $2 \mathrm{D}$ MFI co-crystal microplate exhibits a long-wavelength laser with a high $Q$ value ( 1800) at $\sim 580 \mathrm{~nm}$ (Fig. 11d, e). The MFC microplates of different sizes also show FabryPérot-type microcavity effects, verified by the lasing spectra (Fig. 11f), and the simulated electric field intensity distribution characterization (inset in Fig. 11f). Remarkably, this is the first time that laser oscillation is realized through alleviating the intermolecular CT and modulating the energy-level structure in the organic halogen-bonded co-crystals. However, the stimulated emission of halogen-bonded co-crystal materials remains challenging due to the difficulty in designing and tuning the four-level system for the population inversion.

We developed an approach to form organic halogenbonded co-crystal that transforms the nonlasing micro-

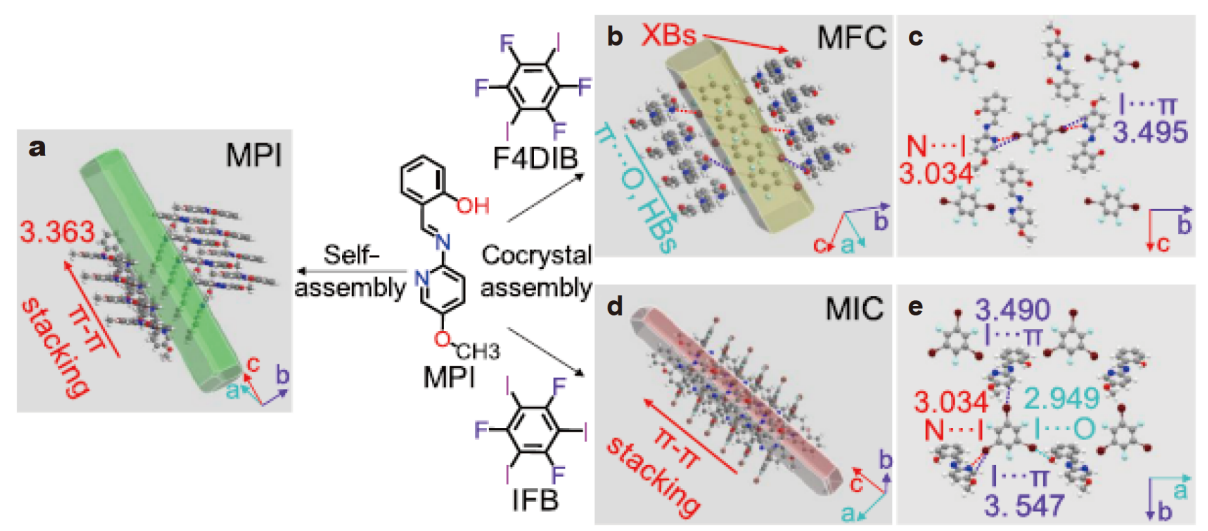

Figure 10 (a, b, d) The speculative growth pattern of MPI, MFC and MIC. (c, e) The halogen bond structures in the co-crystals. Reprinted with permission from Ref. [98]. Copyright 2018, American Chemical Society. 

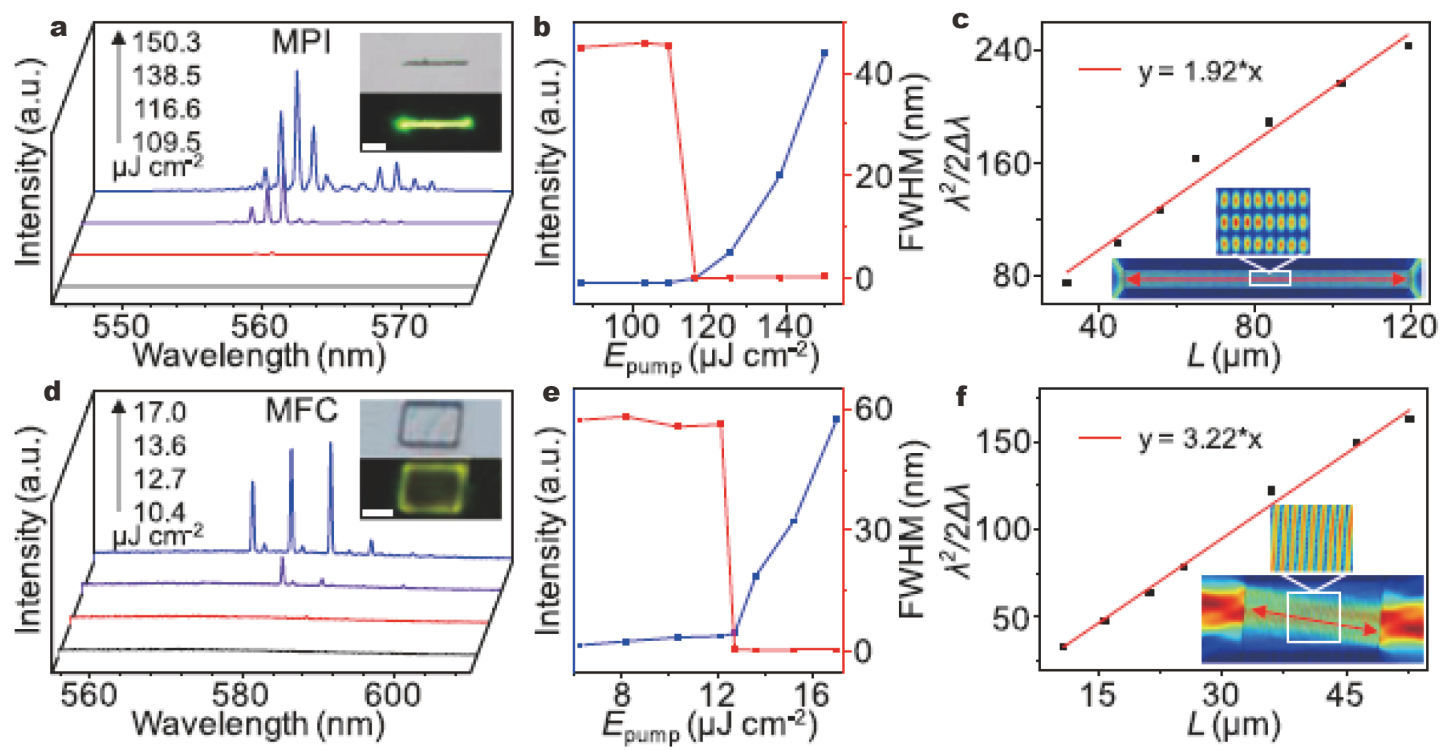

Figure 11 (a, d) Optically pumped lasing measurements for the single MPI wire and MFC plate. Insets: Bright-field and PL images for microwire and microplate. Scale: 20 and $5 \mu \mathrm{m}$. (b, e) Power-dependent profiles (blue) and fwhm (red) of the MPI and MFC. (c, f) Plots and fitted curves of $\lambda^{2} / 2 \Delta \lambda \nu s$. the length of the co-crystal microwires and microplates. Insets: simulated electric field intensity distributions. Reprinted with permission from Ref. [98]. Copyright 2018, American Chemical Society.

crystals to the lasing co-crystals [99]. The as-prepared $o$ BCB microcrystals exhibit a green-emissive $1 \mathrm{D}$ microrod structure, which belongs to the triclinic crystalline lattice and grows along the [001] direction (Fig. 12b-d). Using the halogen bond between $\mathrm{BFC}$ and $o-\mathrm{BCB}$, the $o$-BCB$\mathrm{BFC}$ co-crystal microrods are synthesized, which exhibits a blue-emission and monocline crystalline structure growing along the [010] direction (Fig. 12f-h). The addition of the BFC co-former increases the intermolecular distance between $o$-BCB from 3.06 to $7.10 \AA$ in the $o$ BCB-BFC co-crystal.

The optically pumped lasing characterization reveals that as the excitation intensity attains the maximum value of $20.2 \mu \mathrm{J} \mathrm{cm}^{-2}$, the PL intensity is enhanced, while fwhm remains at $80 \mathrm{~nm}$ (Fig. 13a). This indicates the absence of lasing action for $o$ - $\mathrm{BCB}$ microwires. Relatively, the $o$ BCB-BFC co-crystals exhibit excellent lasing emission. As the excitation intensity increases from 0.8 to $4.1 \mu \mathrm{Jm}^{-2}$, the $o$-BCB-BFC co-crystals show a broad blue emission centered at $450 \mathrm{~nm}$. When the excitation intensity exceeds $4.4 \mu \mathrm{J} \mathrm{cm}^{-2}$, a series of peaks located at $447 \mathrm{~nm}$ appear and fwhm narrows from 22 to $0.4 \mathrm{~nm}$ (Fig. 13b), indicating the occurrence of lasing oscillation. In summary, with the aid of the XB between $\mathrm{BFC}$ and $o-\mathrm{BCB}$, the intermolecular space of the nearby $o$-BCB molecule shortens from 3.06 to $7.10 \AA$, which prevents the excimer in the $o$-BCB microcrystals and offers a true four-level energy level system in $o$-BCB-BFC co-crystals. This organic halogen-bonded co-crystal strategy expands the molecular options for solid-state laser-gain materials.

\section{Nonlinear optics}

Nonlinear optics (NLO) materials based on organic micro/nanocrystals have several practical applications in optoelectronics, such as frequency conversion, electrooptic modulation, and optical parametric oscillation $[100,101]$. However, the structure-property relation, especially for the photophysical and NLO properties, remains unclear in the reported organic halogen-bonded co-crystals [102-104], which hinders the further rational design and fabrication of the co-crystals for optoelectronic devices. In this context, Zhu et al. [105] designed two organic halogen-bonded co-crystals and investigated the molecule-packing-property relation. In the research, Npe and Spe were used as the halogen acceptor, because of their unique $\pi$-conjugated system and the strong ha-

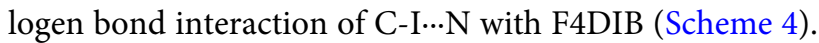
These co-crystals display unique spectra and photophysical properties as compared with the single-component crystals.

The researchers compared the intermolecular interactions, self-assembling behavior, morphology, and crystal structure, as well as the spectroscopic, photophysical, and NLO properties, and found that the strategy of halogen- 

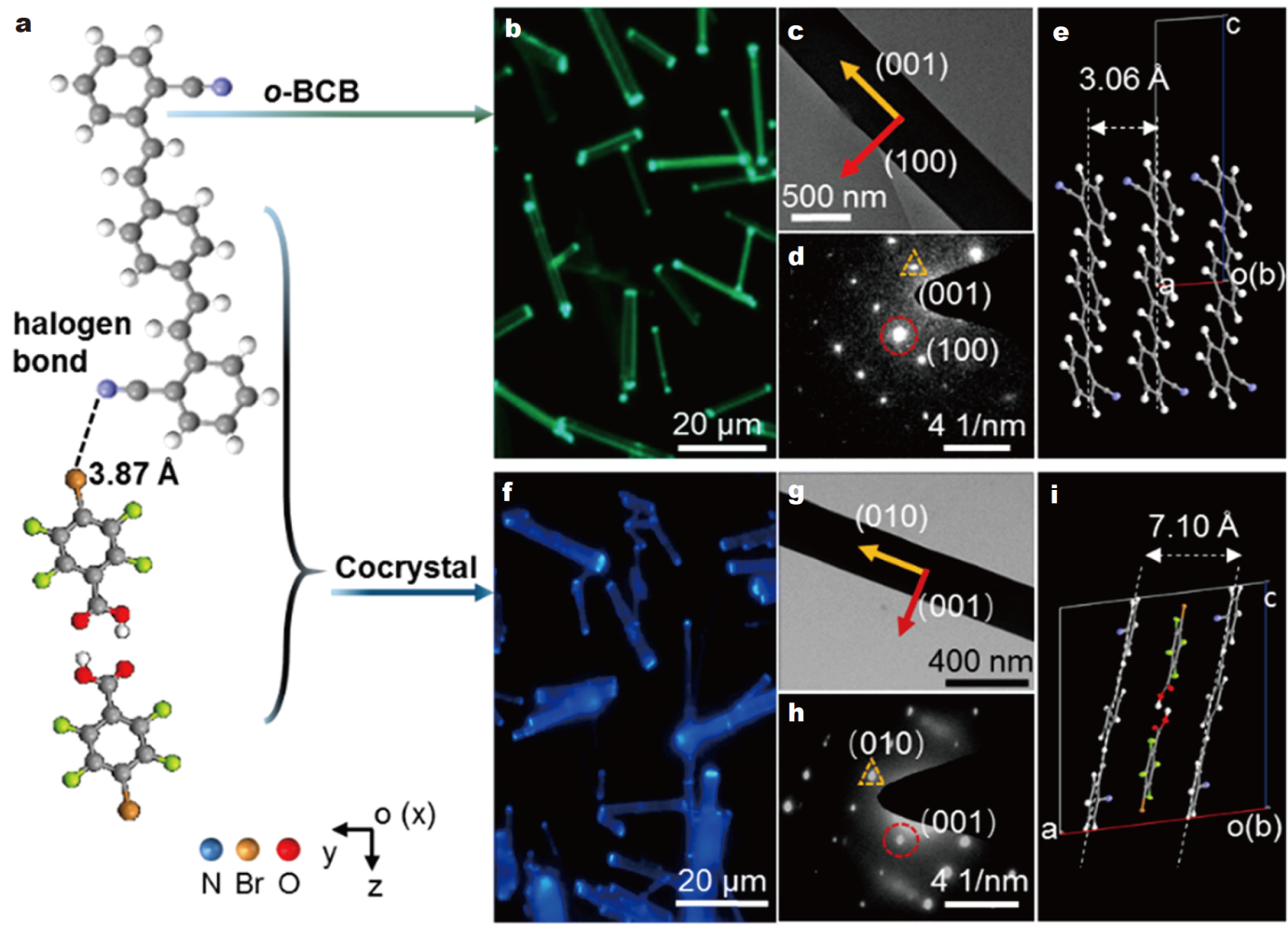

Figure 12 (a) Halogen-bonded co-crystal of $o$-BCB-BFC. (b, f) Fluorescence microscopy images of the $o$-BCB microcrystals and $o$-BCB-BFC cocrystals. (c, g) TEM images of $o$-BCB microrod and $o$-BCB-BFC co-crystal microrod. (d, h) The corresponding SAED pattern. (e) Molecular stacking pattern in $o$-BCB crystal and $o$-BCB-BFC co-crystal. Reprinted with permission from Ref. [99]. Copyright 2019, American Chemical Society.
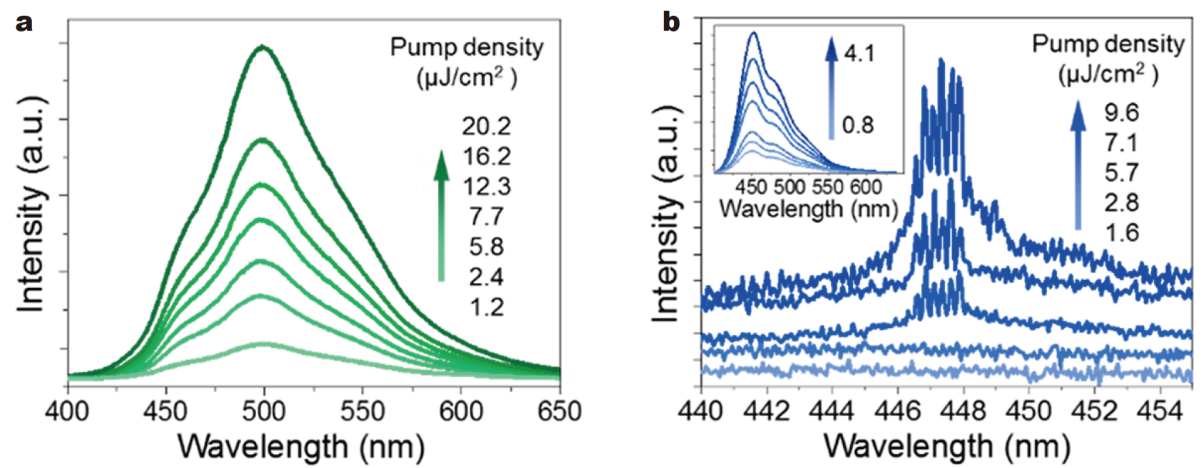

Figure 13 PL spectra obtained by exciting at varing energies for (a) one $o$-BCB microrod and (b) one $o$-BCB-BFC co-crystal microrod. Inset of (b): PL spectra of $o$-BCB-BFC co-crystal microrod with exciting energies below the lasing threshold. Reprinted with permission from Ref. [99]. Copyright 2019, American Chemical Society.

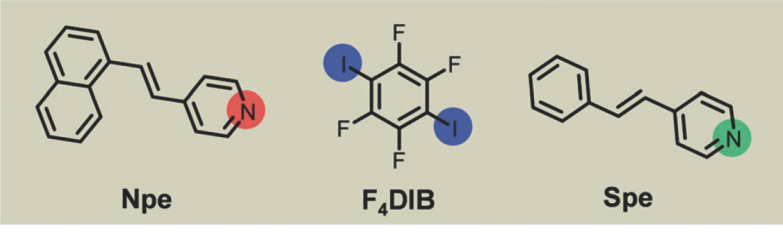

Scheme 4 Molecular structures of Npe, $\mathrm{F}_{4} \mathrm{DIB}$, and Spe. Reprinted with permission from Ref. [105]. Copyright 2016, Wiley-VCH. bonded co-crystals can modulate the NLO properties. The NLO properties of Npe, Spe crystals, and Npe.F4DIB co-crystal (NFC) were investigated independently. As shown in Fig. 14a, as the power of excitation laser increases, the PL intensity of the Npe crystal at $\lambda=420 \mathrm{~nm}$ is enhanced. The fitted linear correlation between the PL intensity and excitation power, as shown in Fig. 14b, demonstrates the two-photon absorption (TPA) process. 

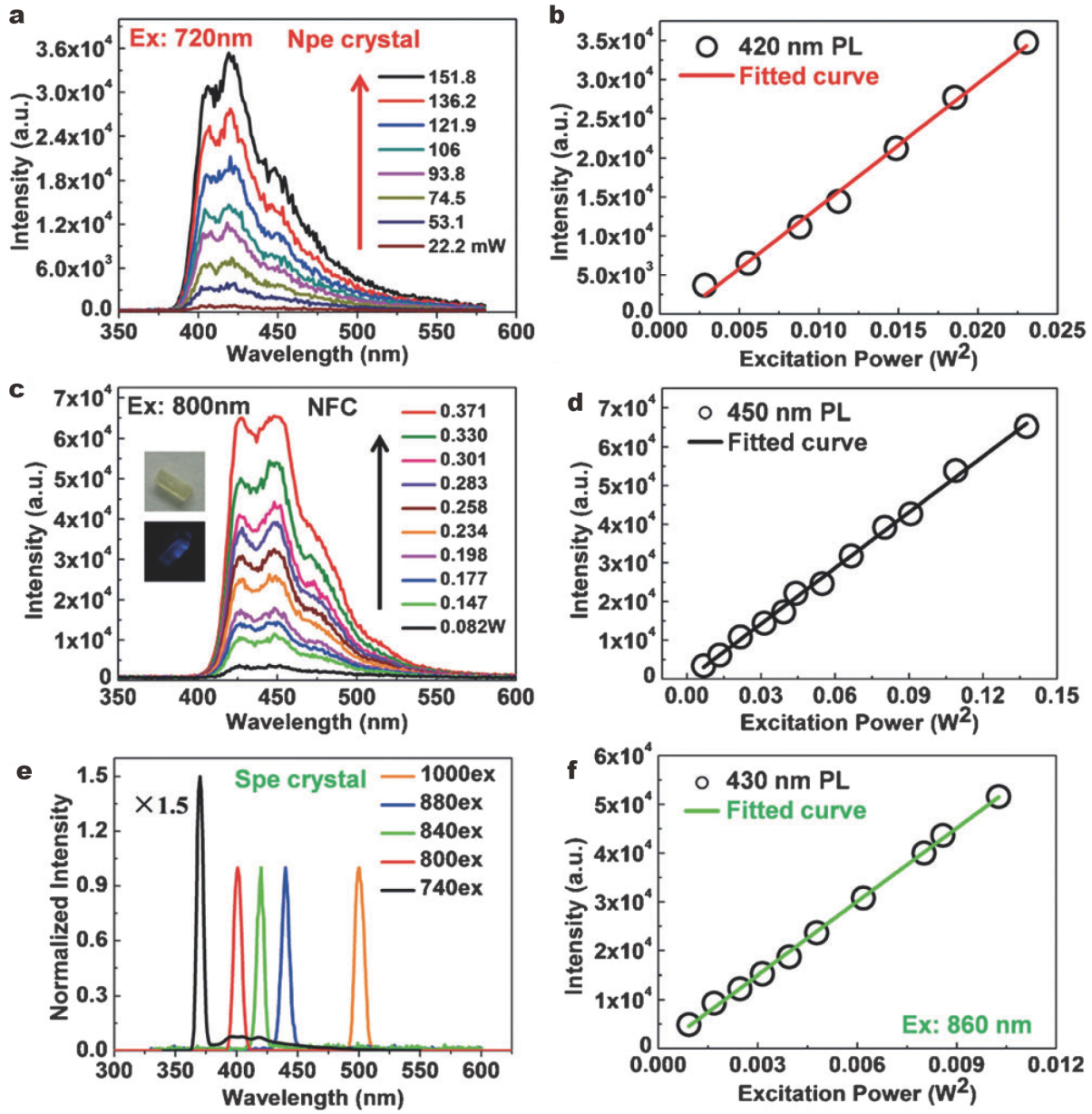

Figure 14 (a, c, e) The up-conversion PL spectra for Npe crystal, NFC co-crystal and Spe crystal. (b, d, f) The fitted linear correlation between the PL intensity and excitation power for Npe crystal, NFC co-crystal and Spe crystal. Reprinted with permission from Ref. [105]. Copyright 2016, Wiley$\mathrm{VCH}$.

Similarly, the linear relation for NFC co-crystals indicates the TPA process while excited with $\lambda_{\mathrm{ex}}=720-840 \mathrm{~nm}$ (Fig. 14c, d). The TPA process in NFC is attributable to the supramolecular electronic polarization, which results in the expansion of D- $\pi$-A configuration. Moreover, the Spe crystals show second harmonic generation (SHG) properties when excited by $\lambda_{\text {ex }}=740-1000 \mathrm{~nm}$. Fig. 14e shows that when Spe is excited with the $740 \mathrm{~nm}$ laser, a weak and sharp peak at $370 \mathrm{~nm}$ appears, and a fitted linear correlation between the PL intensity and the excitation laser is obtained (Fig. 14f). These suggest that the $\mathrm{D}-\mathrm{A}$ structure of the Spe molecule bearing intramolecular electronic polarization and the non-centrosymmetric media results in SHG properties of the Spe crystal. Thus, $\mathrm{XB}$ is beneficial to the NLO properties of the organic cocrystals, which can be advantageous in tuning the optical nonlinearities at the molecule level.

\section{Photonic logic gate}

Optical logic gates with multiple input/output channels have potential applications in optical components for integrated organic photonics $[106,107]$. 2D organic cocrystals with a regular morphology and isotropic/anisotropic molecular stacking pattern result in directionoriented asymmetric photon transport, and hence, can be used as a microscale optical logic gate $[108,109]$. Owing to the anisotropic DPEpe-F4DIB co-crystals, which display direction-oriented asymmetric optical waveguide characteristics, we employ DPEpe-F4DIB co-crystals in the optical logic gate with multiple inputs and output channels, as shown in Fig. 15a1-a8 (the excited laser $\lambda_{\mathrm{ex}}=$ $375 \mathrm{~nm}$ ) [90]. The PL intensity ratios of the four output terminals of the eight typical excitation positions in the same crystal are normalized and divided into four regions correspondingly, as shown in Fig. 15b. The information 

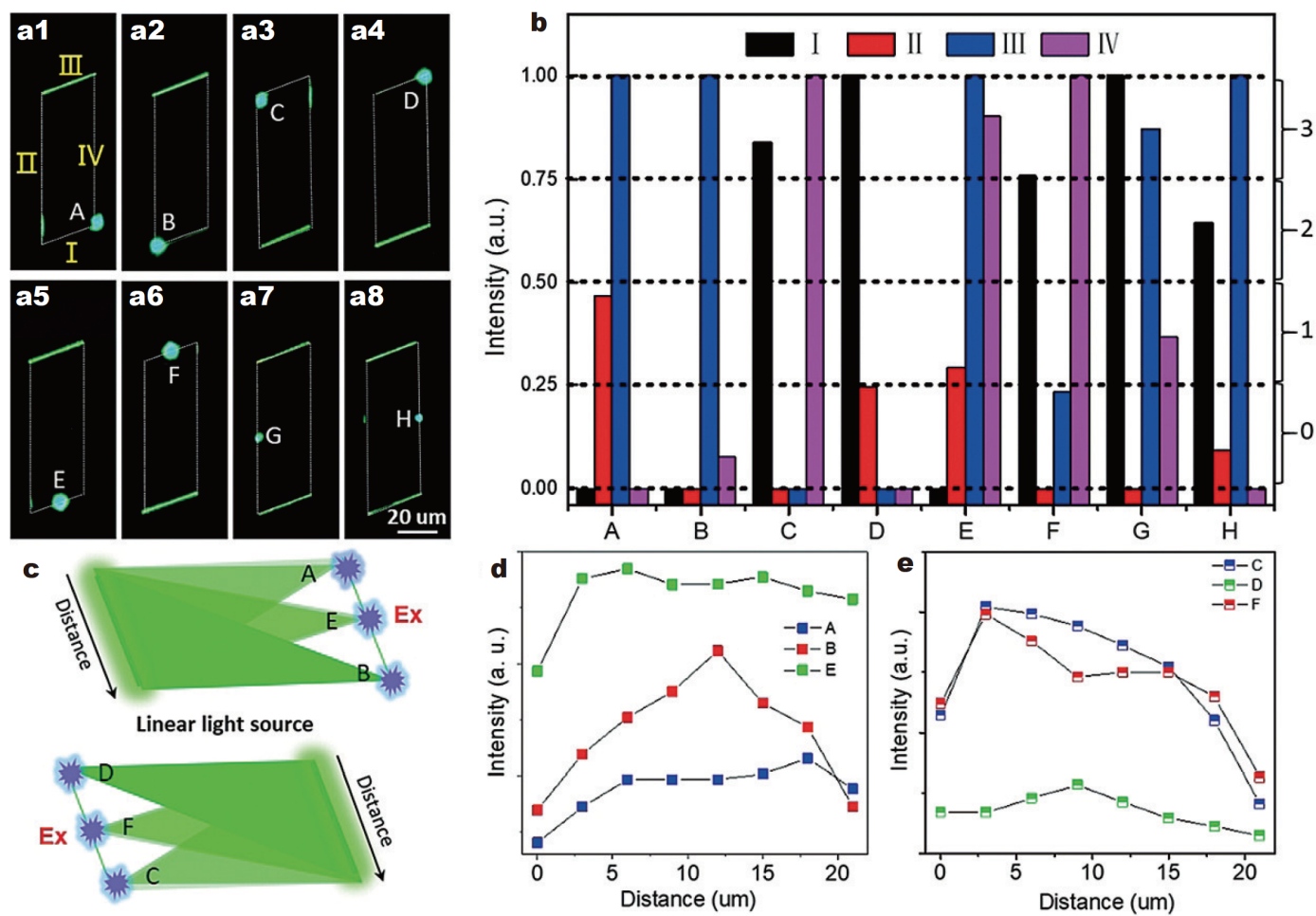

Figure 15 (a1-a8) Fluorescence microscopy images for DPEpe-F4DIB co-crystal excited at eight typical sites with $375 \mathrm{~nm}$ laser beam. (b) The ratios of the intensity of four edges corresponding to the eight exciting sites in one co-crystal microplate. (c) Representation of the microcell edge and the exciting sites at the opposite edges. (d, e) Spatially resolved PL of the microcell edge corresponding to (c). Reprinted with permission from Ref. [90]. Copyright 2018, Wiley-VCH.

codes of $(0,1,3,0)$ can be read through the four output terminals, whereas the input position is located on the lower-right vertex (Fig. 15a, b). The same mechanism is applicable to the other input positions. On the other hand, accurate microcell emission properties are obtained by decomposing the luminescence edge of the organic crystal into eight parts along the [100] direction (Fig. 15c). The microcell emission intensity on the luminescence edge is related to the distance between each typical input position on the other side (Fig. 15d, e). Moreover, as the asymmetric photon is transported along the [010] direction, the intensity of the out-coupled light corresponding to the symmetric input site, especially that of $\mathrm{B}$ and $\mathrm{D}$, demonstrates a unidirectional signal transmission performance. The above results indicate that the multi-channel emission of 2D DPEpe-F4DIB co-crystals displays encoding/decoding functions by using different excitation sites as the encryption keys, and a definite emission intensity model serves as the output signal.

\section{Electrical properties}

CT interactions, as well as CT exciton dynamics, between the donor and acceptor groups in organic co-crystals play an important role in optoelectronic devices. As aforementioned, XBs have been confirmed to be a CT interaction where atoms with lone electron pairs, such as $\mathrm{N}, \mathrm{O}$, and $S$, delocalize to the halogen atom acceptor $\left(n \rightarrow \sigma^{*}\right)$ $[38,43]$. Thus, organic halogen-bonded co-crystals are considered as the $\sigma$-complex. However, only a few studies have been conducted on CT interactions in organic halogen-bonded co-crystals $[110,111]$, which has hindered further rational design and fabrication of organic optoelectronic devices. Zhu et al. [112] conducted an electrical investigation on the organic halogen-bonded co-crystals. They synthesized two types of organic co-crystals with distinct molecular packing modes and systematically investigated the "assembly-structure-CT interaction". They demonstrated that the CT interactions can be tuned via the crystal engineering strategy in organic halogen-bonded co-crystals.

In that study, the selected co-formers are IFB, transBpe, and F4DIB (Scheme 5). Two co-crystals of Bpe-IFB and Bpe-F4DIB were synthesized via solution drop-casting. The electrical conductivities of the obtained co- 

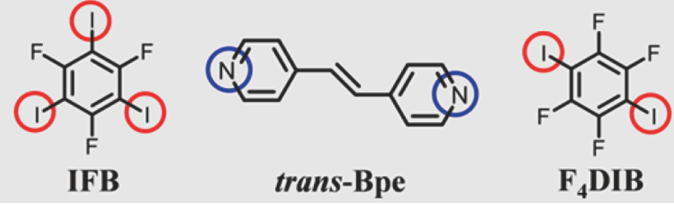

Scheme 5 The molecule structures for Bpe, IFB and F4DIB. Reprinted with permission from Ref. [112]. Copyright 2015, American Chemical Society.

crystals were determined by using a two-terminal microscale device. Each of the BIC microwires shows a typical $I-V$ characteristic curve, as shown in Fig. 16a, and the linear correlation of the $I-V$ behavior obeys ohm's law (Fig. 16b). The electric conductivity $(\sigma)$ of BIC co-crystals is obtained to be $1.42 \times 10^{6} \Omega^{-1} \mathrm{~m}^{-1}$, according to the equation: $\sigma=L / R W H$, where $\sigma$ and $R$ are the electrical conductivity and resistance of the microwire, respectively, and $W, H$, and $L$ denote the width, height, and length of the device channel for the crystal. It indicates that the BIC co-crystals exhibit a quasi-1D semiconductor nature. The BFC co-crystals are obtained to be electrically insulating (Fig. 16c, d), which can be attributed to the mixedstacking structure without CT interactions. Hu's group
[112] attributed the existence of the CT interaction in the separated BIC co-crystals to the $\pi$-electron-rich circumstances in the $\pi-\pi$ stacking Bpe columns, which facilitates the CT interaction in co-crystals. However, in the BFC co-crystals, the Bpe molecules are scattered, thus resulting in a deficiency of $\pi$-electrons, and in turn, a poor CT interaction between Bpe and F4DIB molecules. Therefore, the molecular packing mode in organic halogen-bonded co-crystals has a significant influence on the CT interaction, which in turn affects the optoelectronic properties of the organic co-crystals.

\section{CONCLUSIONS}

$\mathrm{XBs}$ have been confirmed to regulate intermolecular electron transport as well as the PL of organic co-crystals. Thus, organic co-crystals based on them have attracted increasing attention due to their unpredictable and outstanding chemical and physical properties, as well as their potential in optoelectronic applications. In this paper, we have systematically summarized the recent developments in organic halogen-bonded co-crystals with regard to their fabrication methods and optoelectronic properties. Some methods commonly employed in the fabrication of
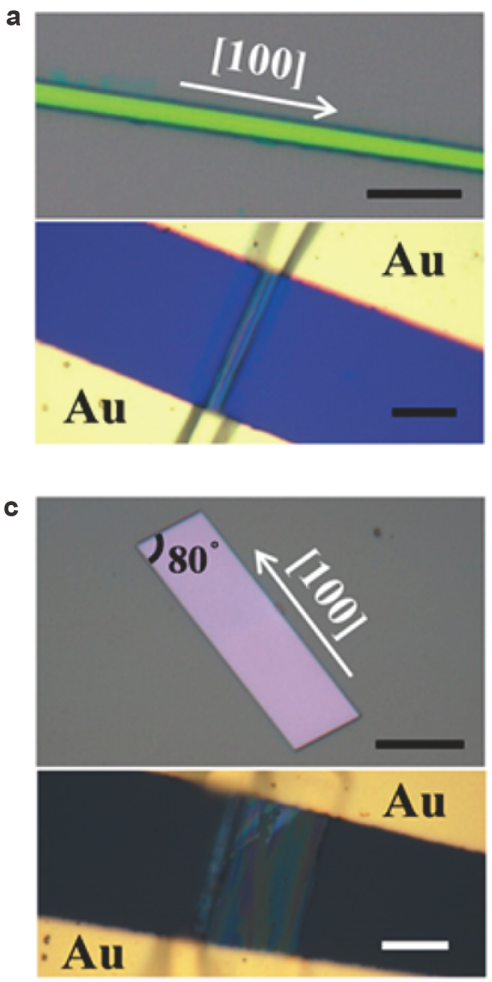
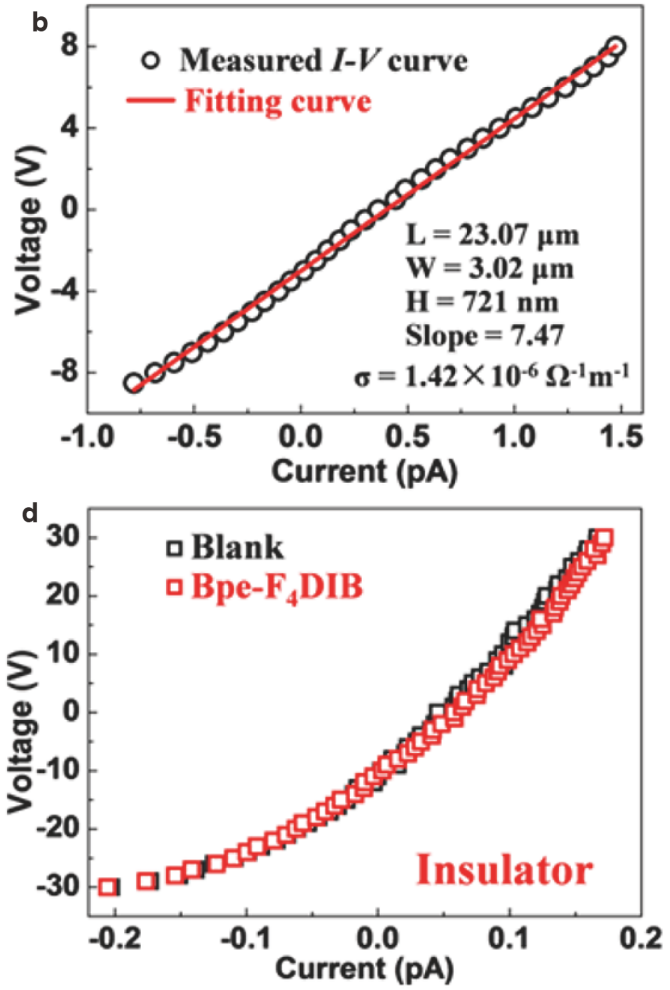

Figure 16 Optical images of (a) BIC co-crystal (upper section) and the device (bottom section), (c) BFC co-crystal (upper section) and microblockbased device (bottom section). The scale bars are $10 \mu \mathrm{m}$. (b, d) $I-V$ curve and corresponding fitted relationship for BIC and BFC co-crystals. Reprinted with permission from Ref. [112]. Copyright 2015, American Chemical Society. 
organic halogen-bonded co-crystals, including solution evaporation, mechanochemical, and liquid-assisted grinding methods, are summarized. Furthermore, the optoelectronic properties are discussed in detail with regards to various applications.

Among the fabrication methods, solution evaporation is the simplest and most efficient method, and is dependent on external conditions such as the solvent, concentration, and temperature. The morphology of the organic co-crystals can be modulated by adjusting the concentration of organic molecules. However, the fabricated micro/nanostructures exhibit random distribution, and thus, cannot form a large-scale array of ordered arrangements. Therefore, there is a need to extend the method to achieve structures with large-scale and highly ordered arrays for practical applications.

For optoelectronic applications, there is still much ambiguity and randomness in the choice of constituent materials for effective co-crystallization with expected functions. Thus, it is imperative to broaden material systems and develop high-performance halogen-bonded co-crystals for the corresponding applications. Taking organic solid-state lasers as an example, a stimulated emission of halogen-bonded co-crystal materials remains a challenge due to the difficulty in tuning of the four-level energy system for population inversion. Thus, the constituent materials, crystal structure, and energy-level structure of halogen-bonded co-crystals need further optimization and development. In addition, efforts are demanded to widen the application of organic halogenbonded co-crystals in electronic fields (such as electrically pumped organic lasers or organic photoelectric detectors), thus boosting the multifunctional development.

In summary, not minding the challenges, there is a promising future for organic halogen-bonded co-crystals. As novel organic semiconductor crystals, we hope that organic halogen-bonded co-crystals would play an important role in the next-generation optoelectronics.

Received 28 February 2020; accepted 7 May 2020; published online 1 July 2020

1 Park SK, Kim JH, Ohto T, et al. Highly luminescent 2D-type slab crystals based on a molecular charge-transfer complex as promising organic light-emitting transistor materials. Adv Mater, 2017, 29: 1701346

2 Briseno AL, Mannsfeld SCB, Ling MM, et al. Patterning organic single-crystal transistor arrays. Nature, 2006, 444: 913-917

3 Zhang J, Jin J, Xu H, et al. Recent progress on organic donoracceptor complexes as active elements in organic field-effect transistors. J Mater Chem C, 2018, 6: 3485-3498

4 Sundar VC, Zaumseil J, Podzorov V, et al. Elastomeric transistor stamps: reversible probing of charge transport in organic crystals. Science, 2004, 303: 1644-1646

5 Park KS, Cho B, Baek J, et al. Single-crystal organic nanowire electronics by direct printing from molecular solutions. Adv Funct Mater, 2013, 23: 4776-4784

6 Pan C, Dong L, Zhu G, et al. High-resolution electroluminescent imaging of pressure distribution using a piezoelectric nanowire LED array. Nat Photon, 2013, 7: 752-758

7 Usman $\mathrm{R}$, Khan $\mathrm{A}$, Wang $\mathrm{M}$, et al. Investigation of chargetransfer interaction in mixed stack donor-acceptor cocrystals toward tunable solid-state emission characteristics. Cryst Growth Des, 2018, 18: 6001-6008

8 Wang $\mathrm{X}$, Liao Q, Kong Q, et al. Whispering-gallery-mode microlaser based on self-assembled organic single-crystalline hexagonal microdisks. Angew Chem Int Ed, 2014, 53: 5863-5867

9 Wang X, Li ZZ, Zhuo MP, et al. Tunable near-infrared organic nanowire nanolasers. Adv Funct Mater, 2017, 27: 1703470

10 Zhang C, Zou CL, Yan Y, et al. Two-photon pumped lasing in single-crystal organic nanowire exciton polariton resonators. J Am Chem Soc, 2011, 133: 7276-7279

11 Mizuno H, Haku U, Marutani Y, et al. Single crystals of 5,5'-bis (4'-methoxybiphenyl-4-yl)-2,2'-bithiophene for organic laser media. Adv Mater, 2012, 24: 5744-5749

12 Guo ZH, Lei T, Jin ZX, et al. T-shaped donor-acceptor molecules for low-loss red-emission optical waveguide. Org Lett, 2013, 15: 3530-3533

13 Chen PZ, Zhang H, Niu LY, et al. A solid-state fluorescent material based on carbazole-containing difluoroboron $\beta$-diketonate: multiple chromisms, the self-assembly behavior, and optical waveguides. Adv Funct Mater, 2017, 27: 1700332

14 Li Z, Kim MH, Wang C, et al. Controlling propagation and coupling of waveguide modes using phase-gradient metasurfaces. Nat Nanotech, 2017, 12: 675-683

15 Feng L, Xu YL, Fegadolli WS, et al. Experimental demonstration of a unidirectional reflectionless parity-time metamaterial at optical frequencies. Nat Mater, 2013, 12: 108-113

16 Fang $X$, Yang X, Yan D. Vapor-phase $\pi-\pi$ molecular recognition: a fast and solvent-free strategy towards the formation of cocrystalline hollow microtube with $1 \mathrm{D}$ optical waveguide and upconversion emission. J Mater Chem C, 2017, 5: 1632-1637

$17 \mathrm{Li} \mathrm{J}$, Zhou K, Liu J, et al. Aromatic extension at 2,6-positions of anthracene toward an elegant strategy for organic semiconductors with efficient charge transport and strong solid state emission. J Am Chem Soc, 2017, 139: 17261-17264

18 Capelli R, Toffanin S, Generali G, et al. Organic light-emitting transistors with an efficiency that outperforms the equivalent light-emitting diodes. Nat Mater, 2010, 9: 496-503

19 Dinelli F, Capelli R, Loi M, et al. High-mobility ambipolar transport in organic light-emitting transistors. Adv Mater, 2006, 18: $1416-1420$

20 McCarthy MA, Liu B, Donoghue EP, et al. Low-voltage, lowpower, organic light-emitting transistors for active matrix displays. Science, 2011, 332: 570-573

21 Hu Y, Ma X, Zhang Y, et al. Detection of amines with fluorescent nanotubes: applications in the assessment of meat spoilage. ACS Sens, 2016, 1: 22-25

22 Stockman MI. Nanoplasmonic sensing and detection. Science, 2015, 348: 287-288

23 McNeil SK, Kelley SP, Beg C, et al. Cocrystals of 10-methylphenthiazine and 1,3-dinitrobenzene: implications for the optical 
sensing of TNT-based explosives. ACS Appl Mater Interfaces, 2013, 5: 7647-7653

24 Schneider HJ. Binding mechanisms in supramolecular complexes. Angew Chem Int Ed, 2009, 48: 3924-3977

25 Zhang J, Gu P, Long G, et al. Switching charge-transfer characteristics from p-type to n-type through molecular "doping" (cocrystallization). Chem Sci, 2016, 7: 3851-3856

26 Desiraju GR. Crystal engineering: from molecule to crystal. J Am Chem Soc, 2013, 135: 9952-9967

27 Huang C, Li Y, Wang N, et al. Progress in research into 2D graphdiyne-based materials. Chem Rev, 2018, 118: 7744-7803

$28 \mathrm{Li} \mathrm{J}$, Jiu T, Duan C, et al. Improved electron transport in $\mathrm{MAPbI}_{3}$ perovskite solar cells based on dual doping graphdiyne. Nano Energy, 2018, 46: 331-337

29 Kuang C, Tang G, Jiu T, et al. Highly efficient electron transport obtained by doping PCBM with graphdiyne in planar-heterojunction perovskite solar cells. Nano Lett, 2015, 15: 2756-2762

$30 \mathrm{Li}$ J, Jiu T, Chen S, et al. Graphdiyne as a host active material for perovskite solar cell application. Nano Lett, 2018, 18: 6941-6947

31 Metrangolo P, Meyer F, Pilati T, et al. Halogen bonding in supramolecular chemistry. Angew Chem Int Ed, 2008, 47: 61146127

32 Messina MT, Metrangolo P, Panzeri W, et al. Intermolecular recognition between hydrocarbon oxygen-donors and perfluorocarbon iodine-acceptors: the shortest $\mathrm{O} \cdots \mathrm{I}$ non-covalent bond. Tetrahedron, 2001, 57: 8543-8550

33 Gagnaux P, Susz BP. Etudes de composés d'addition des acides de LEWIS. XII. Structure, spectre infrarouge et polarisation moléculaire du composé d'addition dioxanne-1,4-diiodacétylène. Helvet Chim Acta, 1960, 43: 948-956

34 Fan E, Vicent C, Geib SJ, et al. Molecular recognition in the solid state: hydrogen-bonding control of molecular aggregation. Chem Mater, 1994, 6: 1113-1117

35 Metrangolo P, Resnati G. Halogen bonding: a paradigm in supramolecular chemistry. Chem Eur J, 2001, 7: 2511-2519

36 Desiraju GR, Ho PS, Kloo L, et al. Definition of the halogen bond (IUPAC Recommendations 2013). Pure Appl Chem, 2013, 85: 1711-1713

37 Gilday LC, Robinson SW, Barendt TA, et al. Halogen bonding in supramolecular chemistry. Chem Rev, 2015, 115: 7118-7195

38 Politzer P, Lane P, Concha MC, et al. An overview of halogen bonding. J Mol Model, 2007, 13: 305-311

39 Colin MM, Gaultier de Claubry H. Sur le combinaisons deL'iode avec les substances vegetales et animales. Ann Chim, 1814, 90: 87-100

40 Guthrie F. XXVIII.-On the iodide of iodammonium. J Chem Soc, 1863, 16: 239-244

41 Hassel O, Hvoslef J, Vihovde EH, et al. The structure of bromine 1,4-dioxanate.. Acta Chem Scand, 1954, 8: 873

42 Hassel O. Structural aspects of interatomic charge-transfer bonding. Science, 1970, 170: 497-502

43 Bent HA. Structural chemistry of donor-acceptor interactions. Chem Rev, 1968, 68: 587-648

44 Clark T, Hennemann M, Murray JS, et al. Halogen bonding: the $\sigma$-hole. J Mol Model, 2007, 13: 291-296

45 Lunghi A, Cardillo P, Messina $\mathrm{T}$, et al. Perfluorocarbonhydrocarbon self-assembling. Thermal and vibrational analyses of one-dimensional networks formed by $\alpha, \omega$-diiodoperfluoroalkanes with K.2.2. and K.2.2.2.. J Fluorine Chem, 1998, 91: 191-194 carbon self-assembling. 1D infinite chain formation driven by nitrogen ‥iodine interactions. J Am Chem Soc, 1998, 120: 82618262

47 Farina A, Meille SV, Messina MT, et al. Resolution of racemic 1,2-dibromohexafluoropropane through halogen-bonded supramolecular helices. Angew Chem Int Ed, 1999, 38: 2433-2436

48 Metrangolo P, Resnati G. Halogen bonding: where we are and where we are going. Cryst Growth Des, 2012, 12: 5835-5838

49 Cavallo G, Metrangolo P, Milani R, et al. The halogen bond. Chem Rev, 2016, 116: 2478-2601

50 Metrangolo P, Neukirch H, Pilati T, et al. Halogen bonding based recognition processes: A world parallel to hydrogen bonding. Acc Chem Res, 2005, 38: 386-395

51 Corradi E, Meille SV, Messina MT, et al. Halogen bonding versus hydrogen bonding in driving self-assembly processes. Angew Chem Int Ed, 2000, 39: 1782-1786

52 Prasang C, Whitwood AC, Bruce DW. Halogen-bonded cocrystals of 4 -( $N, N$-dimethylamino)pyridine with fluorinated iodobenzenes. Cryst Growth Des, 2009, 9: 5319-5326

53 Wang C, Danovich D, Mo Y, et al. On the nature of the halogen bond. J Chem Theor Comput, 2014, 10: 3726-3737

54 Wang W, Wong NB, Zheng W, et al. Theoretical study on the blueshifting halogen bond. J Phys Chem A, 2004, 108: 1799-1805

55 Remsing RC, Klein ML. Halogen bond structure and dynamics from molecular simulations. J Phys Chem B, 2019, 123: 62666273

56 Crihfield A, Hartwell J, Phelps D, et al. Crystal engineering through halogen bonding. 2. Complexes of diacetylene-linked heterocycles with organic iodides. Cryst Growth Des, 2003, 3: 313-320

57 Nguyen HL, Horton PN, Hursthouse MB, et al. Halogen bonding: A new interaction for liquid crystal formation. J Am Chem Soc, 2004, 126: 16-17

58 Gao HY, Shen QJ, Zhao XR, et al. Phosphorescent co-crystal assembled by 1,4 -diiodotetrafluorobenzene with carbazole based on C-I $\cdots \pi$ halogen bonding. J Mater Chem, 2012, 22: 5336-5343

59 Huang Y, Wang Z, Chen Z, et al. Organic cocrystals: Beyond electrical conductivities and field-effect transistors (FETs). Angew Chem Int Ed, 2019, 58: 9696-9711

60 Cinčić D, Friščić T, Jones W. Isostructural materials achieved by using structurally equivalent donors and acceptors in halogenbonded cocrystals. Chem Eur J, 2008, 14: 747-753

61 Zhang P, Bolla G, Qiu G, et al. Halogen bonded cocrystal polymorphs of 1,4-di(4'-pyridyl)-1,3-diacetylene. CrystEngComm, 2017, 19: 4505-4509

62 Yan D, Delori A, Lloyd GO, et al. A cocrystal strategy to tune the luminescent properties of stilbene-type organic solid-state materials. Angew Chem, 2011, 123: 12691-12694

63 Weiss R, Schwab O, Hampel F. Ion-pair strain as the driving force for hypervalent adduct formation between iodide ions and substituted iodobenzenes: Structural alternatives to meisenheimer complexes. Chem Eur J, 1999, 5: 968-974

64 Wang H, Hu RX, Pang X, et al. The phosphorescent co-crystals of 1,4-diiodotetrafluorobenzene and bent 3-ring- $N$-heterocyclic hydrocarbons by $\mathrm{C}-\mathrm{I} \cdots \mathrm{N}$ and $\mathrm{C}-\mathrm{I} \cdots \pi$ halogen bonds. CrystEngComm, 2014, 16: 7942-7948

65 Gao YJ, Li C, Liu R, et al. Phosphorescence of several cocrystals assembled by diiodotetrafluorobenzene and three ring angular diazaphenanthrenes via CI $\cdots \mathrm{N}$ halogen bond. Spectrochim Acta Part A-Mol Biomol Spectr, 2017, 173: 792-799 
$66 \mathrm{Xu}$ J, Liu X, Lin T, et al. Synthesis and self-assembly of difunctional halogen-bonding molecules: A new family of supramolecular liquid-crystalline polymers. Macromolecules, 2005, 38: 3554-3557

67 Lei YL, Liao LS, Lee ST. Selective growth of dual-color-emitting heterogeneous microdumbbells composed of organic chargetransfer complexes. J Am Chem Soc, 2013, 135: 3744-3747

68 Bolton O, Lee K, Kim HJ, et al. Activating efficient phosphorescence from purely organic materials by crystal design. Nat Chem, 2011, 3: 205-210

69 Yan D, Delori A, Lloyd GO, et al. A cocrystal strategy to tune the luminescent properties of stilbene-type organic solid-state materials. Angew Chem Int Ed, 2011, 50: 12483-12486

$70 \mathrm{Wu}$ JJ, Li ZZ, Zhuo MP, et al. Tunable emission color and morphology of organic microcrystals by a "cocrystal" approach. Adv Opt Mater, 2018, 6: 1701300

71 Friscic T, Jones W. Recent advances in understanding the mechanism of cocrystal formation via grinding. Cryst Growth Des, 2009, 9: 1621-1637

72 Wang Y, Zhu W, Dong H, et al. Organic cocrystals: new strategy for molecular collaborative innovation. Top Curr Chem (Z), 2016, 374: 83

73 Zhang J, Liu G, Zhou Y, et al. Solvent accommodation: Functionalities can be tailored through co-crystallization based on 1:1 coronene- $\mathrm{F}_{4} \mathrm{TCNQ}$ charge-transfer complex. ACS Appl Mater Interfaces, 2017, 9: 1183-1188

74 Liao Q, Fu H, Yao J. Waveguide modulator by energy remote relay from binary organic crystalline microtubes. Adv Mater, 2009, 21: 4153-4157

75 Zhuo MP, Tao YC, Wang XD, et al. Rational synthesis of organic single-crystalline microrods and microtubes for efficient optical waveguides. J Mater Chem C, 2018, 6: 9594-9598

76 Cincic D, Friscic T, Jones W. A Stepwise mechanism for the mechanochemical synthesis of halogen-bonded cocrystal architectures. J Am Chem Soc, 2008, 130: 7524-7525

77 d'Agostino S, Grepioni F, Braga D, et al. Tipping the balance with the aid of stoichiometry: room temperature phosphorescence versus fluorescence in organic cocrystals. Cryst Growth Des, 2015, 15: 2039-2045

78 Braga D, Maini L, Grepioni F. Mechanochemical preparation of co-crystals. Chem Soc Rev, 2013, 42: 7638-7648

79 Wang X, Zhou Y, Lei T, et al. Structural-property relationship in pyrazino[2,3-g]quinoxaline derivatives: morphology, photophysical, and waveguide properties. Chem Mater, 2010, 22: 37353745

80 Friščić $\mathrm{T}$, Trask AV, Jones $\mathrm{W}$, et al. Screening for inclusion compounds and systematic construction of three-component solids by liquid-assisted grinding. Angew Chem Int Ed, 2006, 45: 7546-7550

81 Lei YL, Jin Y, Zhou DY, et al. White-light emitting microtubes of mixed organic charge-transfer complexes. Adv Mater, 2012, 24: 5345-5351

82 Chandrasekhar N, Mohiddon MA, Chandrasekar R. Organic submicro tubular optical waveguides: self-assembly, diverse geometries, efficiency, and remote sensing properties. Adv Opt Mater, 2013, 1: 305-311

83 Liu H, Lu Z, Zhang Z, et al. Highly elastic organic crystals for flexible optical waveguides. Angew Chem Int Ed, 2018, 57: 84488452

84 Zhang C, Zhao YS, Yao J. Optical waveguides at micro/nanoscale based on functional small organic molecules. Phys Chem Chem Phys, 2011, 13: 9060-9073

85 Zhao Y, Xu J, Peng A, et al. Optical waveguide based on crystalline organic microtubes and microrods. Angew Chem Int Ed, 2008, 47: 7301-7305

86 Dong R, Pfeffermann M, Liang $\mathrm{H}$, et al. Large-area, free-standing, two-dimensional supramolecular polymer single-layer sheets for highly efficient electrocatalytic hydrogen evolution. Angew Chem Int Ed, 2015, 54: 12058-12063

87 Zhang Z, Song X, Wang S, et al. Two-dimensional organic single crystals with scale regulated, phase-switchable, polymorphismdependent, and amplified spontaneous emission properties. J Phys Chem Lett, 2016, 7: 1697-1702

88 Heng L, Wang X, Tian D, et al. Optical waveguides based on single-crystalline organic micro-tiles. Adv Mater, 2010, 22: 47164720

89 Chandrasekhar N, Chandrasekar R. Reversibly shape-shifting organic optical waveguides: formation of organic nanorings, nanotubes, and nanosheets. Angew Chem Int Ed, 2012, 51: 35563561

90 Zhuo MP, Tao YC, Wang XD, et al. 2D organic photonics: an asymmetric optical waveguide in self-assembled halogen-bonded cocrystals. Angew Chem, 2018, 130: 11470-11474

91 Zhao YS, Peng A, Fu H, et al. Nanowire waveguides and ultraviolet lasers based on small organic molecules. Adv Mater, 2008, 20: $1661-1665$

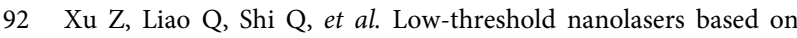
slab-nanocrystals of $\mathrm{H}$-aggregated organic semiconductors. Adv Mater, 2012, 24: OP216-OP220

93 Zhang W, Yao J, Zhao YS. Organic micro/nanoscale lasers. Acc Chem Res, 2016, 49: 1691-1700

94 Huang $\mathrm{MH}$, Mao S, Feick $\mathrm{H}$, et al. Room-temperature ultraviolet nanowire nanolasers. Science, 2001, 292: 1897-1899

95 Wang $\mathrm{X}, \mathrm{Li} \mathrm{H}, \mathrm{Wu} \mathrm{Y}$, et al. Tunable morphology of the selfassembled organic microcrystals for the efficient laser optical resonator by molecular modulation. J Am Chem Soc, 2014, 136: 16602-16608

96 Hill MT, Gather MC. Advances in small lasers. Nat Photon, 2014, 8: 908-918

97 Wang X, Li ZZ, Li SF, et al. Near-infrared organic single-crystal lasers with polymorphism-dependent excited state intramolecular proton transfer. Adv Opt Mater, 2017, 5: 1700027

98 Chu M, Qiu B, Zhang W, et al. Tailoring the energy levels and cavity structures toward organic cocrystal microlasers. ACS Appl Mater Interfaces, 2018, 10: 42740-42746

$99 \mathrm{Yu} \mathrm{Y,} \mathrm{Li} \mathrm{ZZ,} \mathrm{Wu} \mathrm{JJ,} \mathrm{et} \mathrm{al.} \mathrm{Transformation} \mathrm{from} \mathrm{nonlasing} \mathrm{to}$ lasing in organic solid-state through the cocrystal engineering. ACS Photonics, 2019, 6: 1798-1803

100 Rao SM, Batra AK, Lal RB, et al. Mixed methyl-(2,4-dinitrophenyl)-aminopropanoate: 2-methyl-4-nitroaniline crystal-a new nonlinear optical material. J Appl Phys, 1991, 70: 6674-6678

101 Yang Z, Mutter L, Stillhart M, et al. Large-size bulk and thin-film stilbazolium-salt single crystals for nonlinear optics and $\mathrm{THz}$ generation. Adv Funct Mater, 2007, 17: 2018-2023

102 Bosshard C, Wong MS, Pan F, et al. Self-assembly of an acentric co-crystal of a highly hyperpolarizable merocyanine dye with optimized alignment for nonlinear optics. Adv Mater, 1997, 9: 554-557

103 Wuest JD. Co-crystals give light a tune-up. Nat Chem, 2012, 4: $74-75$ 
Goetz KP, Vermeulen D, Payne ME, et al. Charge-transfer complexes: new perspectives on an old class of compounds. J Mater Chem C, 2014, 2: 3065-3076

105 Zhu W, Zhu L, Sun L, et al. Uncovering the intramolecular emission and tuning the nonlinear optical properties of organic materials by cocrystallization. Angew Chem Int Ed, 2016, 55: 14023-14027

106 Andreasson J, Pischel U, Straight SD, et al. All-photonic multifunctional molecular logic device. J Am Chem Soc, 2011, 133: 11641-11648

107 Fu Y, Hu X, Lu C, et al. All-optical logic gates based on nanoscale plasmonic slot waveguides. Nano Lett, 2012, 12: 5784-5790

108 Montenegro JM, Perez-Inestrosa E, Collado D, et al. A naturalproduct-inspired photonic logic gate based on photoinduced electron-transfer-generated dual-channel fluorescence. Org Lett, 2004, 6: 2353-2355

109 Li Y, Zheng H, Li Y, et al. Photonic logic gates based on control of FRET by a solvatochromic perylene bisimide. J Org Chem, 2007, 72: $2878-2885$

110 Lucassen ACB, Karton A, Leitus G, et al. Co-crystallization of sym-triiodo-trifluorobenzene with bipyridyl donors: Consistent formation of two instead of anticipated three N...I halogen bonds. Cryst Growth Des, 2007, 7: 386-392

111 Walsh RB, Padgett CW, Metrangolo P, et al. Crystal engineering through halogen bonding: Complexes of nitrogen heterocycles with organic iodides. Cryst Growth Des, 2001, 1: 165-175

112 Zhu W, Zheng R, Zhen Y, et al. Rational design of charge-transfer interactions in halogen-bonded co-crystals toward versatile solidstate optoelectronics. J Am Chem Soc, 2015, 137: 11038-11046

Acknowledgements This work was supported by the National Natural Science Foundation of China (21703148, 21971185, 21403130, 21403129, 21576158, and 21576159), the Natural Science Foundation of Jiangsu Province (BK20170330), the Natural Science Foundation of Shandong Province (ZR2014BQ028 and 2015ZRB01765), the Collaborative Innovation Center of Suzhou Nano Science and Technology (CIC-Nano), the Priority Academic Program Development of Jiangsu Higher Education Institutions (PAPD), and the "111" Project of The State Administration of Foreign Experts Affairs of China.

Author contributions Chen $S$ prepared the figures and wrote the paper. Yin HL and Wu JJ revised the figures. Wang XD proposed and guided the project. Wang XD and Lin HT revised the manuscript. All authors joined the discussion and gave useful suggestions.

Conflict of interest The authors declare no conflict of interest.

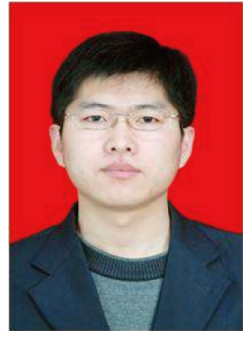

Shuhai Chen received his $\mathrm{PhD}$ degree from the Institute of Coal Chemistry, Chinese Academy of Sciences in 2012. In 2015, he joined the School of Chemical Engineering, Qingdao University of Science and Technology, as a postdoctor. His research interests are in molecular materials, polymer materials, and optoelectronic devices.

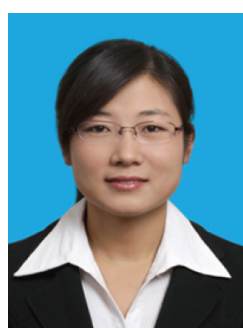

Hongtao Lin is an associate professor in the School of Chemistry and Chemical Engineering, Shandong University of Technology. She received her $\mathrm{PhD}$ degree from the Institute of Chemistry, Chinese Academy of Sciences, in 2013 after she got her MSc degree (2010) in Lanzhou Institute of Chemical Physics, Chinese Academy of Sciences. Her research work includes the design and synthesis of novel organic semiconductors, and fabrication and characterization of organic optoelectronic devices.

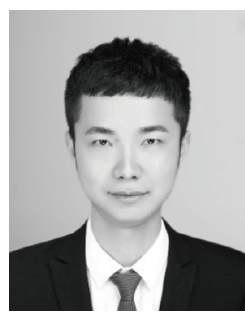

Xue-Dong Wang is an associate professor at the Institute of Functional Nano \& Soft Materials (FUNSOM), Soochow University. He received his Bachelor's degree in chemistry from Lanzhou University in 2011 and his $\mathrm{PhD}$ in physical chemistry from the Institute of Chemistry, Chinese Academy of Sciences in 2016. His research focuses on the fine synthesis of organic micro/ nanocrystals and the organic photonics, including organic solid-state lasers and optical waveguides.

\section{有机卤素键晶体及其在光电子学中的应用}

陈淑海 ${ }^{1,3}$, 尹慧玲 ${ }^{1}$, 吴俊杰 ${ }^{2}$, 芴红桃 ${ }^{*}$, 王雪东 ${ }^{2 *}$

摘要 有机卤素共晶是由两个或者更多不同组分通过碳卤键构成 的. 因其多样化的化学物理特性, 及在有机光电子学领域的潜在应 用价值, 有机卤素共晶引起了研究者的广泛关注. 本综述文章中, 我 们简单总结了有机卤键共晶的制备方法和在光电子器件领域应用 的最新进展. 此外, 我们还讨论了目前有机卤键共晶的研究方向及 面临的技术挑战, 同时对有机卤键共晶的进一步研究和应用前景 进行了展望. 